\title{
Exchange Bias in Thin Films-An Update
}

\author{
Tomasz Blachowicz ${ }^{1}$ (D) and Andrea Ehrmann ${ }^{2, *(D)}$ \\ 1 Institute of Physics-Center for Science and Education, Silesian University of Technology, \\ ul. Konarskiego 22B, 44-100 Gliwice, Poland; tomasz.blachowicz@polsl.pl \\ 2 Faculty of Engineering and Mathematics, Bielefeld University of Applied Sciences, Interaktion 1, \\ 33619 Bielefeld, Germany \\ * Correspondence: andrea.ehrmann@fh-bielefeld.de
}

check for

updates

Citation: Blachowicz, T.; Ehrmann, A. Exchange Bias in Thin Films-An Update. Coatings 2021, 11, 122. https://doi.org/10.3390/coatings 11020122

Received: 31 December 2020

Accepted: 20 January 2021

Published: 22 January 2021

Publisher's Note: MDPI stays neutral with regard to jurisdictional claims in published maps and institutional affiliations.

\begin{abstract}
The exchange bias (EB) is an effect occurring in coupled ferromagnetic/antiferromagnetic materials of diverse shapes, from core-shell nanoparticles to stacked nanostructures and thin films. The interface coupling typically results in a horizontal—often also vertical—shift of the hysteresis loop, combined with an increased coercivity, as compared to the pure ferromagnet, and the possibility of asymmetric hysteresis loops. Several models have been developed since its discovery in 1956 which still have some drawbacks and some unexplained points, while exchange bias systems are at the same time being used in hard drive read heads and are part of highly important elements for spintronics applications. Here, we give an update of new theoretical models and experimental findings regarding exchange bias phenomena in thin films during the last years, including new material combinations in which an exchange bias was found.
\end{abstract}

Keywords: exchange bias (EB); ferromagnet; antiferromagnet; coercivity; coercive field; hysteresis loop shift; asymmetric hysteresis loop; domain state model

\section{Introduction}

After its discovery in 1956, using Co/CoO core-shell particles [1], the exchange bias (EB) effect has been investigated in detail by many research groups. Typically, it occurs when a ferromagnet (FM) and an antiferromagnet (AFM) are in contact, and the system is cooled from high temperatures through the Néel temperature (the magnetic ordering temperature) of the AFM in an external magnetic field. In a naïve concept, this process freezes the AFM spins, in this way adding an "internal" magnetic field to the external one which leads to a horizontal shift of the FM hysteresis loop. However, other correlated phenomena, such as a vertical shift of the loop and an often occurring asymmetry of the hysteresis loop [2], cannot be explained by this most simple model.

While first studies concentrated on relatively simple FM/AFM combinations, such as the aforementioned $\mathrm{Co} / \mathrm{CoO}[3,4], \mathrm{Fe} / \mathrm{FeF}_{2}[5,6]$, or $\mathrm{Fe} / \mathrm{MnF}_{2}[7,8]$, which already exhibited quite interesting effects for the different materials, nowadays more complicated materials are under investigations, such as $\mathrm{Fe} / \mathrm{LaAlO}_{3}$ [9] or $\mathrm{Pr}_{0.67} \mathrm{Sr}_{0.33} \mathrm{MnO}_{3} / \mathrm{SrTiO}_{3}$ [10], and more sophisticated structures, such as nanostructures [11-13] or multi-layer sandwiches [14,15].

Still, at the same time, new models are being developed to understand the exchange bias effect, which is already intensively used in magnetic data storage, qualitatively and quantitatively for all these different material combinations and structures.

Here, we give an update of recent experimental and theoretical research in the area of exchange bias. To avoid the additional impact of an in-plane component of the shape anisotropy in nanostructured or nanoparticle systems, we concentrate in this review on thin film samples, where the shape anisotropy often results in the magnetic moments being oriented in-plane. 


\section{Properties of Exchange Bias Systems}

Several models were developed during the last decades, aiming at describing the experimentally found effects in exchange bias systems, ideally not only qualitatively, but also quantitatively. Besides the aforementioned shift and a possible asymmetry of the hysteresis loop, a reasonable model should also take into account the following properties.

The exchange bias is temperature-dependent. In the simplest case, the EB field is reduced with increasing temperature and vanishes at the so-called blocking temperature which may be much lower than the Néel temperature of the antiferromagnet for thin AFM layer and can approximately reach the Néel temperature in case of bulk antiferromagnets. Besides, the blocking temperature is influenced by the FM/AFM interface and the structural order in the AFM [16,17]. In more special system, such as a ferromagnet coupled to a spin glass, additional effects may occur, e.g., reversing the sign of the EB directly below the blocking temperature [18].

In many cases, the exchange bias depends on the ferromagnetic layer thickness, often with an antiproportional correlation [16], underlining that the EB is an interface effect. The AFM thickness, on the other hand, may have different impact on the exchange bias, depending on the material system and the technological preparation parameters [16].

The exchange bias can depend on the cooling field intensity; however, the reported dependencies differ. Sometimes, large cooling fields result in a reduction [19] or even a reversal of the exchange bias [20,21]; in other studies, the EB increases with increasing fields, until it saturates [22].

The interface roughness is quite an important parameter, although in most models its influence on the exchange bias is not fully understood, and modeled results are often not consistent with the experiment. Typically, quite flat interfaces of AFM crystals result in a small exchange bias, while rougher interfaces show an increased EB. Oppositely, in many thin film systems the EB is larger for smoother interfaces and reduced by an increased roughness, but can also increase again after a minimum at a middle roughness [2,23]. Other systems show only a dependence on special roughness parameters [24].

The exchange bias not only depends on the surface roughness of the AFM, but also on its crystallinity. Twinned antiferromagnets, containing two sub-lattices with different main orientations, e.g., may exhibit a higher exchange bias than fully epitaxial AFM layers, as shown, among others, for $\mathrm{Fe} / \mathrm{FeF}_{2}$ and $\mathrm{Co} / \mathrm{CoO}[2,25]$. Polycrystalline films often show a larger EB with smaller grain size, while the opposite behavior is also possible, depending on the preparation parameters of the layered systems [16].

Another important factor is the interface orientation. For the often investigated antiferromagnet $\mathrm{FeF}_{2}$, e.g., the spin are oriented in the (110) plane, meaning that they are perpendicularly oriented to the (001) plane. Former studies found a maximum exchange bias in layered systems based on this antiferromagnet for an interface orientation along the (110) plane, a vanishing EB for a (001) interface, and an average exchange bias for a (101) oriented surface [16].

Besides these-often contradictory—findings, there are other well-known effects which can often be found in exchange bias systems. One of them is a maximum of the coercivity near the blocking temperature, e.g., found in $\mathrm{Fe} / \mathrm{FeF}_{2}(110)$ and $\mathrm{Co} / \mathrm{CoO}(110)$, which can be attributed to the $90^{\circ}$ coupling which will be taken into account in some of the models described in the next section. Another one is the so-called training effect which results in a reduction of the exchange bias during subsequent measurements without new field cooling [26-28], occurring even in systems beyond the conventional FM/AFM coupled systems [29].

\section{Modeling the Exchange Bias}

Explaining all the aforementioned phenomena qualitatively and quantitatively by a model is obviously difficult, especially as different effects are found in different material systems or even in identical material systems, prepared in different ways. This is why after the first intuitive models, several other emerged, aiming at explaining as many 
experimentally observed effects as possible in a physically reasonable way. Naturally, with new experimental findings, new models are necessary, sometimes only valid for very special cases, sometimes explaining a broad range of phenomena at least qualitatively.

To start with an intuitive model, it can be assumed that uncompensated interface spins of the AFM (i.e., the AFM spins whose summation vectors are not zero) couple to the FM. While free uncompensated AFM spins increase the coercivity of the system, pinned uncompensated AFM spins results in an induced unidirectional anisotropy, i.e., the exchange bias [30]. In such models, the exchange bias field can vanish due to roughness which effectively compensates the AFM spins, or it can be overestimated by some orders of magnitude [31].

Néel published a model based on collinear AFM and bulk FM magnetization with uniaxial anisotropy and an uncompensated AFM interface. He found that domains can be created in the equilibrium state which could explain several effects connected with the exchange bias, but on the other hand, the model could not be applied to thin film systems since a bulk FM had to be assumed [32].

Next, the random field model of Malozemoff was published, based on a compensated spin structure and a rough interface, as depicted in Figure 1 [33]. Opposite to the simplest models, however, Malozemoff now allowed domain walls perpendicular to the interface to be formed. This means that in addition to the interface energy, the domain wall energy has to be taken into account [34-36]. In other words: while the interface energy is reduced by forming domains with small net spins corresponding to the favored interface coupling, the domain wall energy is increased by forming domains, so that the final domain formation in the energetically favored state depends on the superposition of these two contributions. The formed domains are frozen at low temperatures, thus supporting an exchange bias. Depending on the surface roughness, sensible values of the exchange bias can be reached within this model.

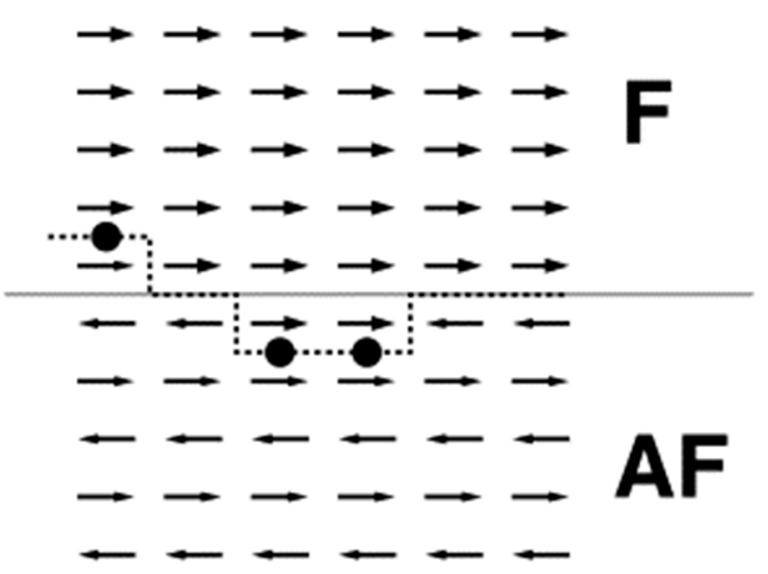

Figure 1. Ferromagnet (F)/antiferromagnet (AF) thin film system with antiferromagnetically coupled interface. The roughness (dashed line) results in frustrated interactions. Reprinted with permission from [33], Copyright 2001 Elsevier.

Mauri's model included also non-collinear orientations of FM and AFM spins (Figure 2) [37]. By this assumption, domains parallel to the interface were enabled. Interestingly, the exchange bias field calculated within this model was the same as in the historical model of Meiklejohn and Bean who used single-domain AFM and FM with a possible in-plane angle between them and calculated the equilibrium by setting the torque on ferromagnetic and antiferromagnetic moments as zero [38]. 


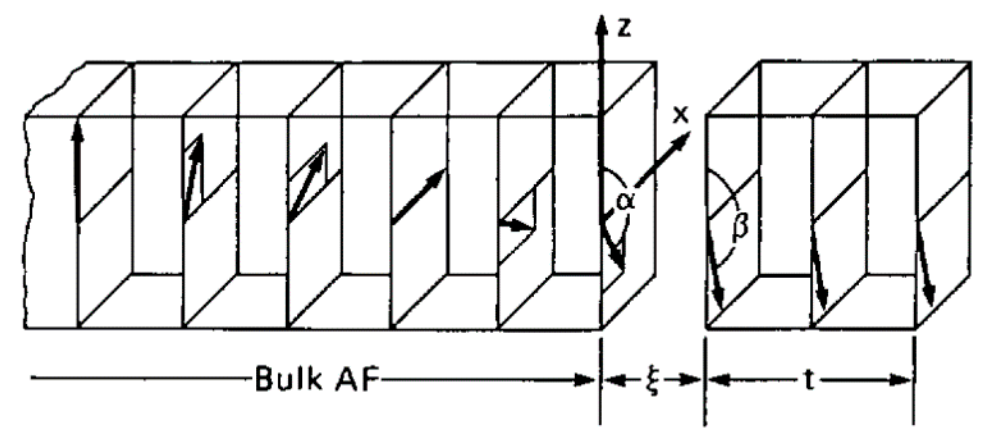

Figure 2. Magnetic model of a bulk antiferromagnet (AFM) (only one sublattice's spins are shown) in contact with a FM thin film. Here, the uniaxial AFM anisotropy is oriented along the positive $\mathrm{z}$-direction, while the external magnetic field is oriented along the negative $\mathrm{z}$-direction. Reprinted with permission from [37], Copyright 2001 AIP Publishing.

Schulthess and Butler used a three-dimensional Heisenberg-type model with singlecrystalline FM and AFM, connected at an even interface with antiferromagnetic coupling. Interestingly, the equilibrium solutions of the Landau-Lifshitz-Gilbert equation revealed states with the ferromagnetic spins being oriented perpendicular to the easy axis of the AFM. If the AFM spins are allowed to show a slight canting, a new uniaxial anisotropy consequently occurred in the ferromagnet. This aforementioned $90^{\circ}$ coupling, however, can only explain the increased coercive fields, but not the exchange bias [39,40]. Defects located at the perfectly flat surface, on the other hand, can indeed result in an exchange bias, with approximately realistic values for a spin surplus in the order of magnitude of $1 \%$, as it was also experimentally found [41,42]. The model of Schulthess and Butler thus shows the importance of interface defects, but cannot derive them theoretically.

For a polycrystalline AFM and a single-crystal FM, Stiles and McMichael modeled the exchange bias in a Heisenberg model, partly including the $90^{\circ}$ coupling. Depending on the interface coupling strength, they found results similar to Mauri, for the case without $90^{\circ}$ coupling, and a strong dependence on the $90^{\circ}$ coupling strength, if this is taken into account [43].

While the aforementioned single-crystal and polycrystalline AFMs had only interface defects, the domain state model is based on introducing defects also in the AFM volume [44-47]. Such an AFM with defects is also called "diluted antiferromagnet" (Figure 3) [48]. Thus, adequate Monte Carlo simulations were performed based on a Heisenberg model, assuming long-range order based on a uniaxial in-plane anisotropy in the FM. The dilution of the upper layer of the AFM could be fixed to investigate the influence of a varying bulk dilution combined with a constant interface roughness. Simulations were performed along a direction slightly different from the easy axis of the ferromagnet.

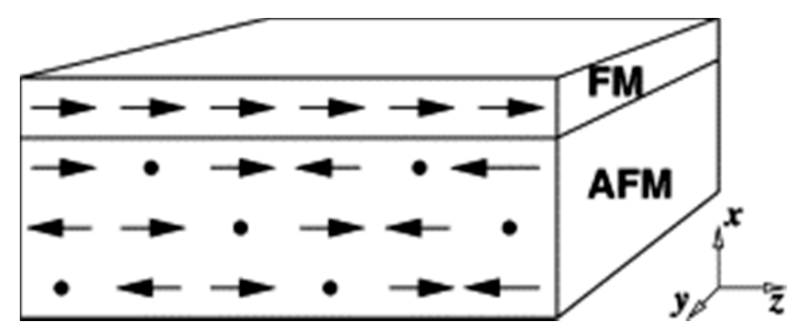

Figure 3. Exchange bias model used in the domain state model, consisting of one ferromagnetic and three diluted antiferromagnetic layers. Reprinted with permission from [48], Copyright 2001 Elsevier.

These simulations showed indeed hysteresis loops which were shifted horizontally and vertically (Figure 4), the latter due to the significant vertical shift of the AFM interface layer (Figure 4 b). Interestingly, the latter occurred even during zero-field cooling, i.e., cool- 
ing without an external magnetic field. The volume magnetization of the AFM, however, showed this vertical shift only after field cooling [49].
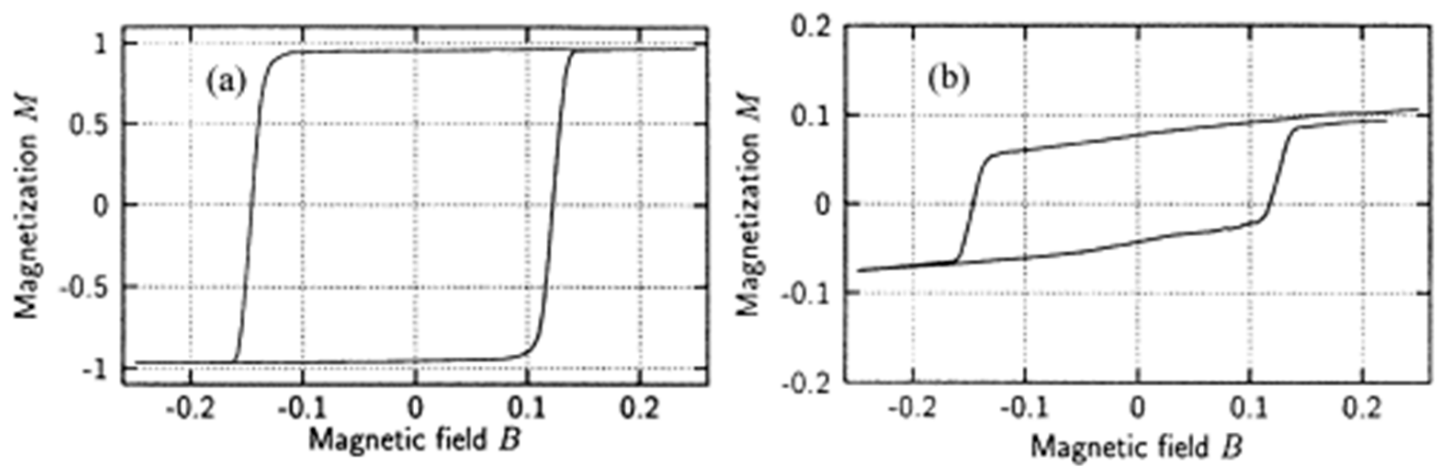

Figure 4. Hysteresis loops of (a) the ferromagnet and (b) the interface layer of the AFM. Reprinted with permission from [48], Copyright 2001 Elsevier.

The authors concluded that the frozen interlayer magnetization of the AFM worked like an additional effective magnetic field on the system, causing the exchange bias effect. The exchange bias was calculated to be proportional to the irreversible domain state magnetization, which corresponds to the vertical shift of the hysteresis loop of the interface layer. The hysteretic behavior of the AFM interface layer and volume was interpreted as the AFM spins switching together with the FM, in this way increasing the coercive fields, while the residual (not switching) part of the AFM interface spins is responsible for the exchange bias.

Another interesting point visible in Figure $4 \mathrm{~b}$ is that the AFM interface layer hysteresis is not closed, suggesting a reduction of the magnetization during a hysteresis loop, which can explain the aforementioned training effect.

It should be mentioned that, opposite to the model of Malozemoff, applying defects only in the interface layer results in the domain state model in vanishing domains in the AFM [44-49].

Another model was suggested by Kiwi et al. who used an AFM with large anisotropy which aimed at simulating $\mathrm{Fe} / \mathrm{FeF}_{2}$ and $\mathrm{Fe} / \mathrm{MnF}_{2}$, especially the compensated (110) interface [50-52]. The large anisotropy in the AFM results in much smaller domain wall widths in the AFM than in the FM [53,54], which on the other hand led to perpendicular FM and AFM spins near the interface and "frozen" spin canting in the AFM interface layer, combined with an incomplete domain wall in the ferromagnet. In this way, they found exchange bias values similar to experimental ones, including even positive exchange bias [52-54].

After the few years around 2000 when several exchange bias models based on different assumptions were suggested, there are still many new models being presented, often taking into account special systems or effects and often based on Monte Carlo or micromagnetic simulations.

Radu et al. extended the model by Meiklejohn and Bean by a spin disorder at the interface to model polycrystalline $\mathrm{Ir}_{17} \mathrm{Mn}_{83} / \mathrm{Co}_{70} \mathrm{Fe}_{30}$ thin film systems [55]. They managed not only simulating the measured longitudinal and transverse hysteresis loops, including sharp transitions of the magnetization reversal processes near the easy axis, but also found some, at first glance, unexpected deviations of the experimental and the simulated EB from the usually assume sinus-like dependence on the angular sample orientation.

Investigating $\mathrm{Mn}_{52} \mathrm{Pd}_{48} / \mathrm{Fe}$ bilayers with soft- $x$-ray magnetic circular dichroism and magnetic reflectivity, Brück et al. found that the $\mathrm{Mn}$ atoms neighboring Fe atoms coupled antiferromagnetically to them so strongly that they completely followed the Fe hysteresis loops, so that these Mn atoms could be regarded as an extension layer of the ferromagnet. 
The next Mn layer, however, exhibited also pinned Mn spins, ferromagnetically coupling to $\mathrm{Fe}$, i.e., aligned antiferromagnetically to the rotating Mn spins [30].

Harres and Geshev published a model for polycrystalline FM/AFM systems. They suggested that for a large enough exchange coupling at the interface, the influence of the AFM results in small domains forming in the FM, while interface grains can be rotatable or fixed. Their model could simulate the non-zero hard axis coercive field (easy and hard axes describe energetically favorable and not favorable orientations of the magnetization), experimentally found in a Co/IrMn film, which was not possible with a model based on a constant rotatable anisotropy, but with the rotatable anisotropy varying along the hysteresis loop, as assumed here [56].

Saha and Victora also investigated polycrystalline biaxial and uniaxial exchange bias systems, based on a micromagnetic simulation in which the ferromagnetic interface grains were coupled to neighboring AFM grains. Using $\mathrm{NiFe} / \mathrm{NiMn}$ data, reasonable values for coercivity and exchange bias were reached. Interestingly, the crystallographic symmetry of the AFM was found to be correlated with the shape of the system's hysteresis loop, i.e., showing asymmetric magnetization reversal for biaxial and symmetric hysteresis loops for uniaxial AFM anisotropy, as well as with the training effect [57].

Another grain-level model was developed by Choo et al. [58]. They simulated a soft magnetic ferromagnet coupled to a hard magnetic antiferromagnet, in which the ferromagnet consisted of strongly exchange-coupled grains in a micromagnetic simulation. As the AFM was assumed to have isolated grains of high anisotropy, its magnetic state was assumed to be dominated by thermally activated processes which were modeled by a kinetic Monte Carlo approach. Their model predicted a peak in the coercivity near the blocking temperature due to instability of the AFM layer, connected with a uniaxial contribution to the interlayer exchange energy superposing the unidirectional exchange bias contribution [58]. Such peaks of the coercive field near the blocking temperature are well known from $\mathrm{Co} / \mathrm{CoO}(110)$ and other systems.

$\mathrm{O}^{\prime}$ Grady et al. also investigated polycrystalline thin films [59]. Summarizing their theoretical work, they found that the EB in such samples is correlated with the thermally stable part of the AFM, as well as with grain size distribution and film thickness. The single AFM grains were found to be independent and to reverse magnetization similarly to the Stoner-Wohlfarth mechanism in ferromagnets. On the other hand, interfacial effects independent from the order in the bulk regions of AFM layer were found. These interface spins showed a tendency to clustering, with these clusters behaving similarly to the aforementioned spin glass states and showing sophisticated interactions with AFM and FM layers [59].

Mangin et al. investigated the ferrimagnetic/ferrimagnetic bilayer system $\mathrm{Gd}_{40} \mathrm{Fe}_{60}$ / $\mathrm{Tb}_{12} \mathrm{Fe}_{88}$ experimentally and by a quantitative model [60], based on unidimensional micromagnetic models for hard/soft magnetic bilayers [61,62]. Under the assumption that the room temperature magnetization configuration inside the TbFe layer is frozen during field cooling, they performed micromagnetic simulations which could well simulate the experimental values of the coercive fields, exchange bias fields and even the vertical loop shift [60]. As they underlined, these experimental results were similar to those found in the more often investigated systems $\mathrm{Fe} / \mathrm{FeF}_{2}$ and $\mathrm{Fe} / \mathrm{FeMn}_{2}$. It should be mentioned that in their model, there was also the possibility of the formation of a domain wall parallel to the interface, as already discussed in other models $[37,50,63]$.

Morales et al. modeled FM/AFM bilayers with very thin FM layers and used a springlike domain structure parallel to the interface to quantitatively simulate the deviation from the inverse proportionality of the EB with the thickness of the ferromagnetic layer [64].

Dimitriadis et al. investigated the exchange bias in core-shell nanoparticles of different shapes by an atomistic model, applying a Heisenberg model with uniaxial anisotropy [65]. Depending on the shape and dimension of the nanoparticles, they found negative exchange bias values especially for spherical particles, which were explained by the highly uncom- 
pensated interface of such spherical nanoparticles, while a cubical particle showed lower exchange bias due to its mostly compensated interfaces [65].

Using a Monte Carlo simulation, Iglesias et al. also modeled core-shell nanoparticles [66]. They found several qualitative agreements with typical experimental findings, e.g., they also modeled, besides the temperature-dependent horizontal shift of the hysteresis loop and broadening of the coercive field, asymmetric hysteresis loops due to different reversal mechanisms in both loop branches and a vertical shift of the loops due to the same reason. In addition, they found a correlation between the net magnetization of the interfacial shell spins and the exchange bias field [66].

Most recently, simulations showed a correlation of the exchange bias with the DzyaloshinskiiMoriya interaction, e.g., in $\mathrm{MnN}$ (bulk)/Fe systems [67], or a coexistence of exchange bias and Dzyaloshinskii-Moriya interaction, like in $\mathrm{Pt} / \mathrm{Co} / \mathrm{IrMn}$ or $\mathrm{Pt} / \mathrm{Co} / \mathrm{FeMn}$ thin films [68] or $\mathrm{Pt} / \mathrm{Co} / \mathrm{Ni}_{x} \mathrm{O}_{y}$ (Figure 5) [69]. Besides, diverse other simulations of new effects and special systems can be found in the literature; dealing, e.g., with the sign reversal of the $\mathrm{EB}$ in $\mathrm{LuFe}_{0.5} \mathrm{Cr}_{0.5} \mathrm{O}_{3}$ ferrite-chromite due to the competition of various Dzyaloshinskii-Moriya interactions [70], voltagecontrolled exchange bias [71], or arbitrarily shaped magnonic nano-waveguides with engineered spin-texture in an exchange-biased bilayer [72].

Trilayer structure
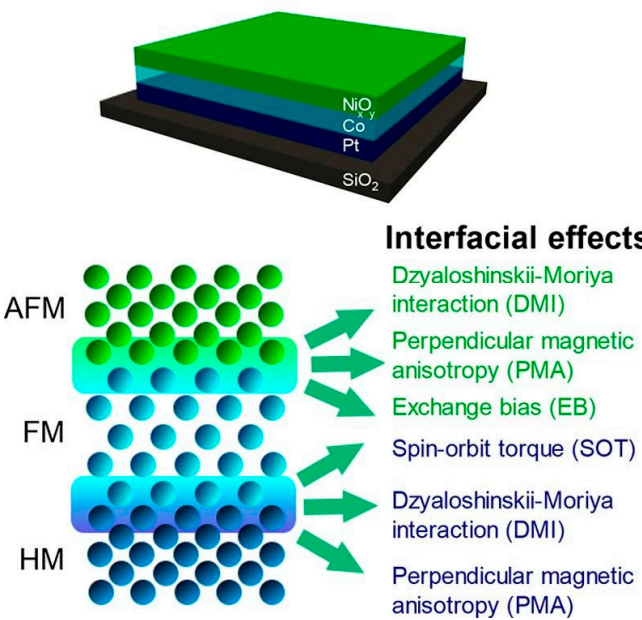

Anisotropy and exchange bias

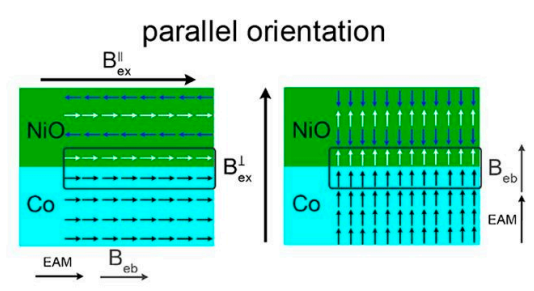

orthogonal orientation

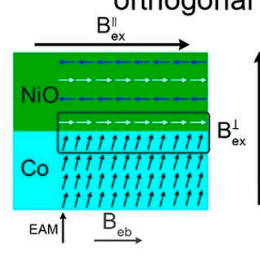

XPS phase analysis
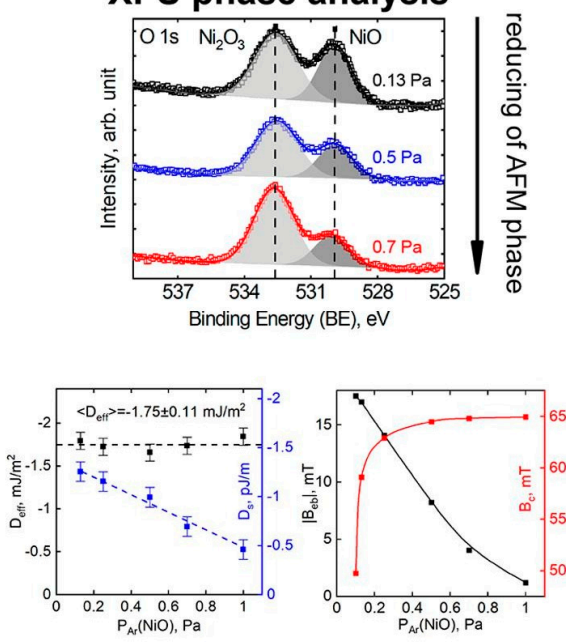

Figure 5. Effects possibly occurring in a trilayer structure of AFM, FM and heavy metal (HM): Dzyaloshinskii-Moriya interaction, exchange bias, spin-orbit torque in the FM and perpendicular magnetic anisotropies in the system $\mathrm{NixOy} / \mathrm{Co} / \mathrm{Pt}$. Reprinted with permission from [69], Copyright 2021 Elsevier.

In the next sections, some newly discovered effects in well-known systems are described, before more complicated thin-film systems will be evaluated.

\section{Traditional Thin Film Systems \\ 4.1. Co/CoO Thin Film Systems}

The exchange bias was originally found in $\mathrm{Co} / \mathrm{CoO}$ core/shell structures, and $\mathrm{Co} / \mathrm{Co}_{3} \mathrm{O}_{4}$ was used as a model system for diluted antiferromagnets in proving the domain state model. This exchange bias system is thus well known for a long time and often used in research. Still, many recent studies work with core/shell nanoparticles [73-76] or, on the other hand, on nanostructures [76-78]. Here, however, we concentrate on research on thin-film systems.

Sharma et al. recently investigated thin $\mathrm{Co} / \mathrm{CoO}$ films deposited on an array of selfassembled polystyrene nanospheres [79]. In this way, they managed combining pure thin layers with an out-of-plane component of the layer, thus modifying the shape anisotropy. Their results showed clear differences between this modified thin film and common thin films, prepared on an even wafer, as depicted in Figure 6 [79]. Clear deviations of the hysteresis loop shape and the exchange bias fields are visible between the different substrates, 
but also for different layer thicknesses. For a layer thickness of $100 \mathrm{~nm}$, only a small EB is visible in both cases, while for a layer thickness of $5 \mathrm{~nm}$, a much larger EB effect can be recognized. Interestingly, even for the thicker Co layer there is a modification of the slope of the hysteresis loop. While the plane reference sample shows a simple nearly square easy-axis loop $(100 \mathrm{~nm}, \mathrm{Si})$, the thick film on polystyrene tends to a combination of easy and hard axis loops which was explained by the film being combined of plane Co dots on top of the nanospheres with the patterns of the narrower regions. For the thin film on polystyrene nanospheres, this effect is much more pronounced, showing a very special shape of the hysteresis loop [79]. Interestingly, this shape was also reported in the literature for $\mathrm{Co} / \mathrm{CoO}$ nanoparticle films [80] and even $\mathrm{Co} / \mathrm{CoO}$ thin films [81], and attributed to a combination of soft and hard magnetic phases, similar to the explanation here based on the shape anisotropy with different orientations [79]. It should be mentioned that a small asymmetry of the hysteresis loop was even observed in $\mathrm{Co} / \mathrm{CoO}$ slightly above the blocking temperature [82], which may be correlated with the disorder of the AFM near the blocking temperature, mentioned in the model of Choo et al. [58].

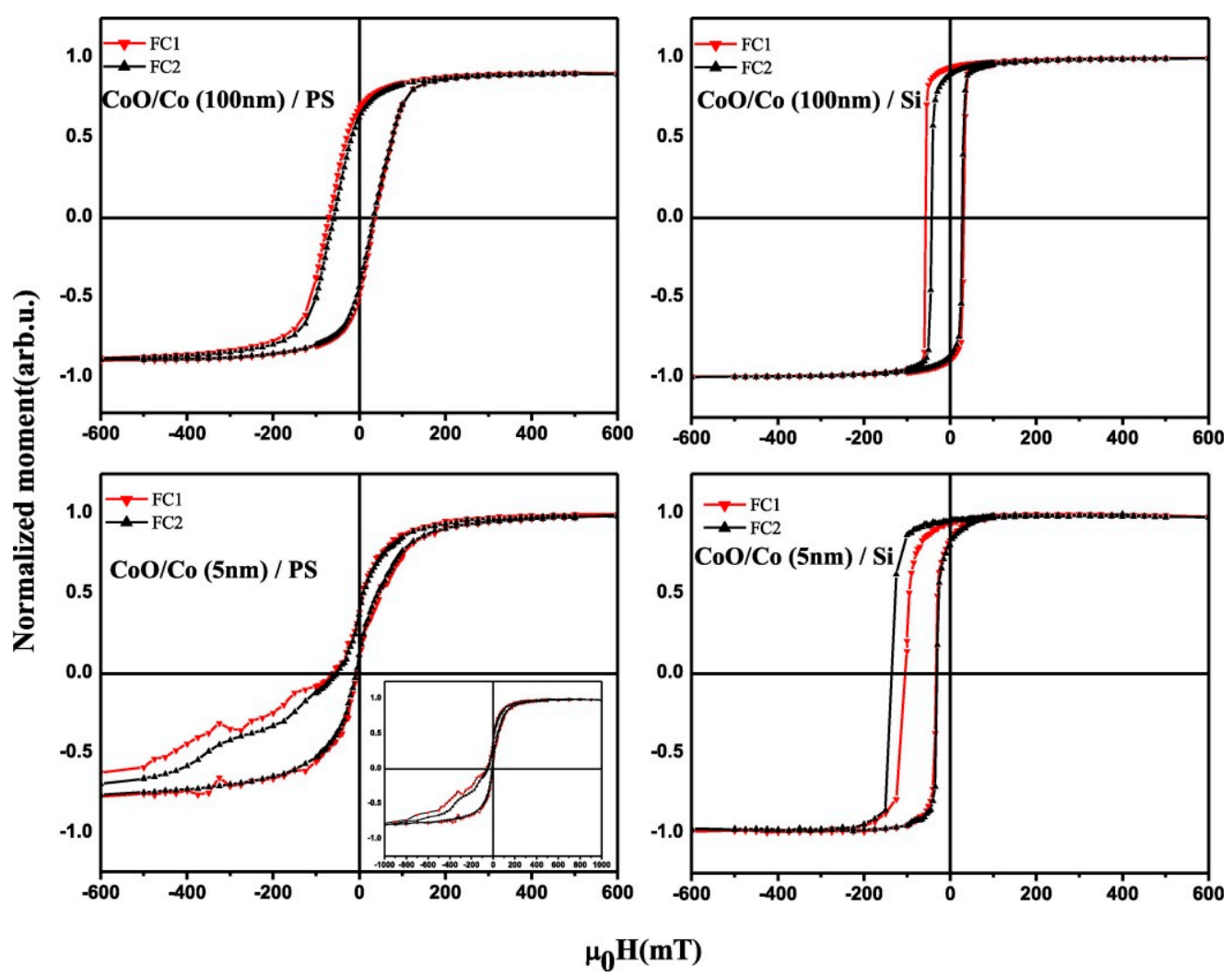

Figure 6. Measurements at $T=5 \mathrm{~K}$ (i.e., far below the blocking temperature near room temperature) on $\mathrm{Co} / \mathrm{CoO}$ thin films (thickness $5 \mathrm{~nm}$ or $100 \mathrm{~nm}$ ) on polystyrene (PS) nanospheres or plane Si substrates, respectively. Reprinted with permission from [79], Copyright 2020 Elsevier.

A similar idea was investigated by Jochum et al. who deposited $\mathrm{Co} / \mathrm{CoO}$ on a pattered substrate, decorated with gold nanoparticles, under an oblique angle, in this way modifying the magneto-crystalline as well as the shape anisotropy [83]. In this way, they prepared a lower nanostructured layer of $\mathrm{Co}$, on top of which oxidized Co in smaller bubbles was found. This very special granular thin-film system showed hysteresis loops with strong exchange bias after field cooling to $1.8 \mathrm{~K}$, exhibiting an asymmetry of the curves unlike the one depicted in Figure 6, but with a kink at small negative fields which was attributed to the interaction of a Co/CoO EB system with a purely ferromagnetic Co layer [83].

An interesting combination of thin-film technology with nanostructuring was reported by Ovejero et al. [84]. They used a $\mathrm{Co} / \mathrm{CoO}$ target for sputter-coating a kapton substrate 
with a Co layer with intrinsic pores around which the Co was oxidized to CoO. They found a double-hysteresis loop for zero-field cooling to $5 \mathrm{~K}$ and a clear exchange bias shift for field cooling in a field of min. $5 \mathrm{kOe}$, combined with the expected decrease of the exchange bias and the coercivity with increasing temperature. In this way, porous $\mathrm{Co} / \mathrm{CoO}$ exchange systems with high thickness could be prepared [84].

Hussain and Reddy used Kerr microscopy to investigate thermal and athermal training effects in a polycrystalline $\mathrm{Co} / \mathrm{CoO}$ bilayer system [85]. Generally, a thermal training effect is attributed to thermally activated gradual de-pinning of frozen AFM moments during hysteresis cycling, while the athermal training effect stems from an AFM interface layer reorientation from the metastable state during field cooling. As expected, they found a reduction of the EB with increasing FM layer thickness, but also a weaker training effect, which was attributed to a decreasing disorder at the interface with increasing FM thickness. For a small FM thickness, they observed an athermal training effect which changes the domain structure and decreases the domain size of the FM layers, while for thicker FM layers, a thermal training effect was observed which did not significantly influence the FM domains [85]. The same group investigated the layer thickness of the $\mathrm{AFM}$ in $\mathrm{Co} / \mathrm{CoO}$ thin film systems and found similar $\mathrm{EB}$, but different training effects for different AFM layer thicknesses between 5 and $12.3 \mathrm{~nm}$ [86]. With increasing AFM thickness, the interface relaxed more slowly during training, i.e., the thicker AFM was more stable, while the thinner ones showed a deviation of the interface magnetization orientation from the direction after field cooling [86].

Kumar et al. investigated the influence of the surface roughness in polycrystalline $\mathrm{Co} / \mathrm{CoO}$ bilayers by preparing samples which were annealed for different durations to increase the interface roughness [87]. They found a decrease of the EB by more than $50 \%$ during increasing the roughness by approximately a factor 2 (Figure 7), which was attributed to weakening the effective spin coupling at the interface by increasing interface disorder [87].

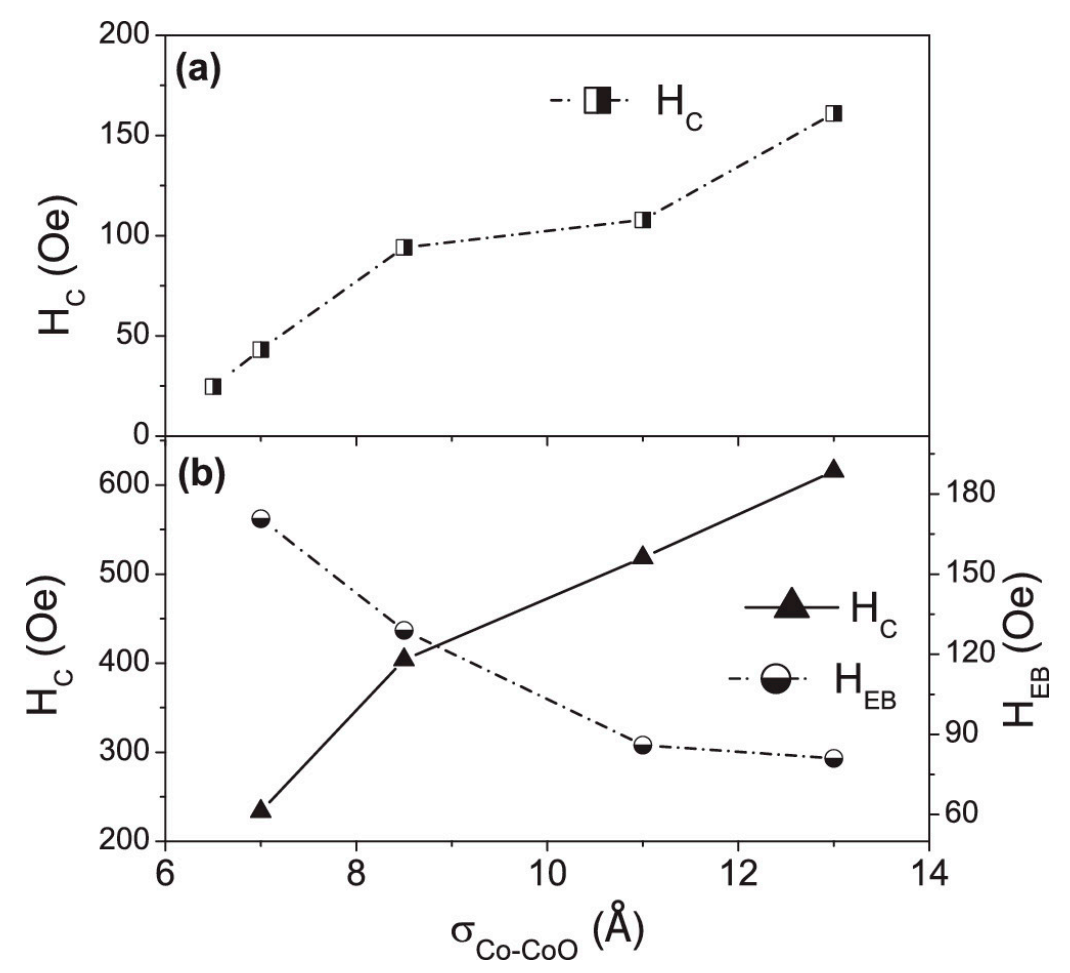

Figure 7. Increase of the coercive field $\mathrm{H}_{\mathrm{C}}(\mathbf{a})$ at room temperature and (b) after field cooling in $1.5 \mathrm{kOe}$ to $153 \mathrm{~K}$, and (b) decrease of the exchange bias field $\mathrm{H}_{\mathrm{EB}}$ under the same conditions with increasing interface roughness. Reprinted with permission from [87], Copyright 2016 AIP Publishing. 
Dobrynin et al. investigated the positive EB and the aforementioned increase in the coercive field near the blocking temperature in $\mathrm{Co} / \mathrm{CoO}$ samples with different AFM grain sizes and prepared a model for this special behavior [88]. They modeled the FM as singledomain and the AFM consisting of one layer on top of the FM, with the uncompensated AFM grain magnetization being oriented collinear to the cooling field direction. With this model, they managed indeed showing a slight positive EB directly below the blocking temperature. Opposite to their experimental results, however, they found a strong variation of the magnitude of the positive $\mathrm{EB}$ and the temperature range in which it occurs with only small differences in the grain size, while both effects were approximately independent from the grain size in the experiment (Figure 8). This finding was interpreted by assuming that the AFM parts involved in the reversal must have identical sizes for each sample, independent from the grain sizes. This, on the other hand, led to the assumption that only single AFM spins were responsible for the reversal, i.e., the reversal of the AFM had to occur on an atomic level. On the other hand, the peak of the coercivity which was observed in their experiments, as well as in several other experimental studies, was attributed to an increased number of mobile interface AFM moments whose energy barriers added to the energy barrier of the FM layer during magnetization reversal [88].

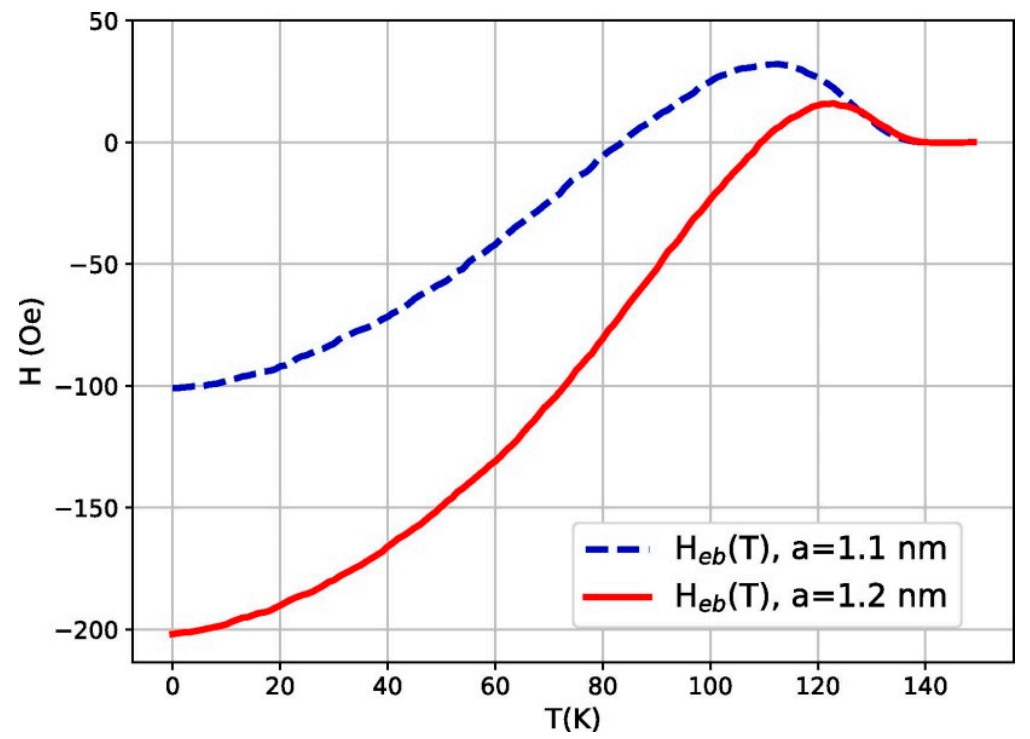

Figure 8. Temperature-dependent exchange bias, modeled for slightly different grain sizes. Reprinted with permission from [88], Copyright 2021 Elsevier.

Finally, due to the importance of the system $\mathrm{Co} / \mathrm{CoO}$, a few examples of other geometries shall be mentioned here. Singh et al., e.g., studied the exchange bias in $\mathrm{Co} / \mathrm{CoO} / \mathrm{Co}$ trilayer systems. They found two-step rounded hysteresis loops due to different thicknesses and thus different coercive fields of the top layer, as compared to the bottom Co layer, and at low temperatures different asymmetries of the coercive fields for different top layer thicknesses [15].

Using micromagnetic simulations of antiferromagnetic grains in a ferromagnetic matrix, Menéndez et al. showed that the training effect in such granular systems as well as the asymmetry in the hysteresis loop during first reversal are correlated with the perpendicular anisotropy of rotatable interface moments [89].

Nanostructured films from $\mathrm{Co} / \mathrm{CoO}$ core-shell clusters were prepared by Xing et al., who found strong exchange coupling between the Co cores and the CoO shells below a temperature of $175 \mathrm{~K}$, with a small positive EB over approx. $100 \mathrm{~K}$ [74].

As these examples from recent years show, even in the long-known system $\mathrm{Co} / \mathrm{CoO}$ in a simple thin-film form there are still new experimental and theoretical findings pos- 
sible. The next sections will present recent findings in similarly well-known exchange bias systems.

\subsection{Ni/NiO Thin Film Systems}

Exchange bias in $\mathrm{Ni} / \mathrm{NiO}$ thin films was already found in 1967 [32] and afterwards reported, similar to $\mathrm{Co} / \mathrm{CoO}$, for core-shell nanoparticles, nanotubes, and other nanostructures [90-93].

$\mathrm{Ni}$ thin films can be oxidized in air, with the NiO layer thickness depending on the environmental temperature [94]. For a constant annealing temperature of $400{ }^{\circ} \mathrm{C}$ in oxygen atmosphere, on the other hand, Raghavan et al. investigated the influence of the annealing time and found an increase of the Ni crystal size with annealing time for shorter durations which finally saturated [95]. At the same time, the NiO layer grew at the cost of the $\mathrm{Ni}$ layer. Besides, annealing firstly led to a more uniform morphology and a decreased surface roughness, while for longer annealing durations, grains started agglomeration resulting in again increased surface roughness. Interestingly, magnetic force microscopy measurements at room temperature showed a magnetic strip pattern in the sample annealed for $90 \mathrm{~min}$ (Figure 9). This may be correlated with an increased $\mathrm{NiO}(111)$ texture in this special sample, as compared to the $\mathrm{Ni}(200)$ texture found for the other annealing durations [95].
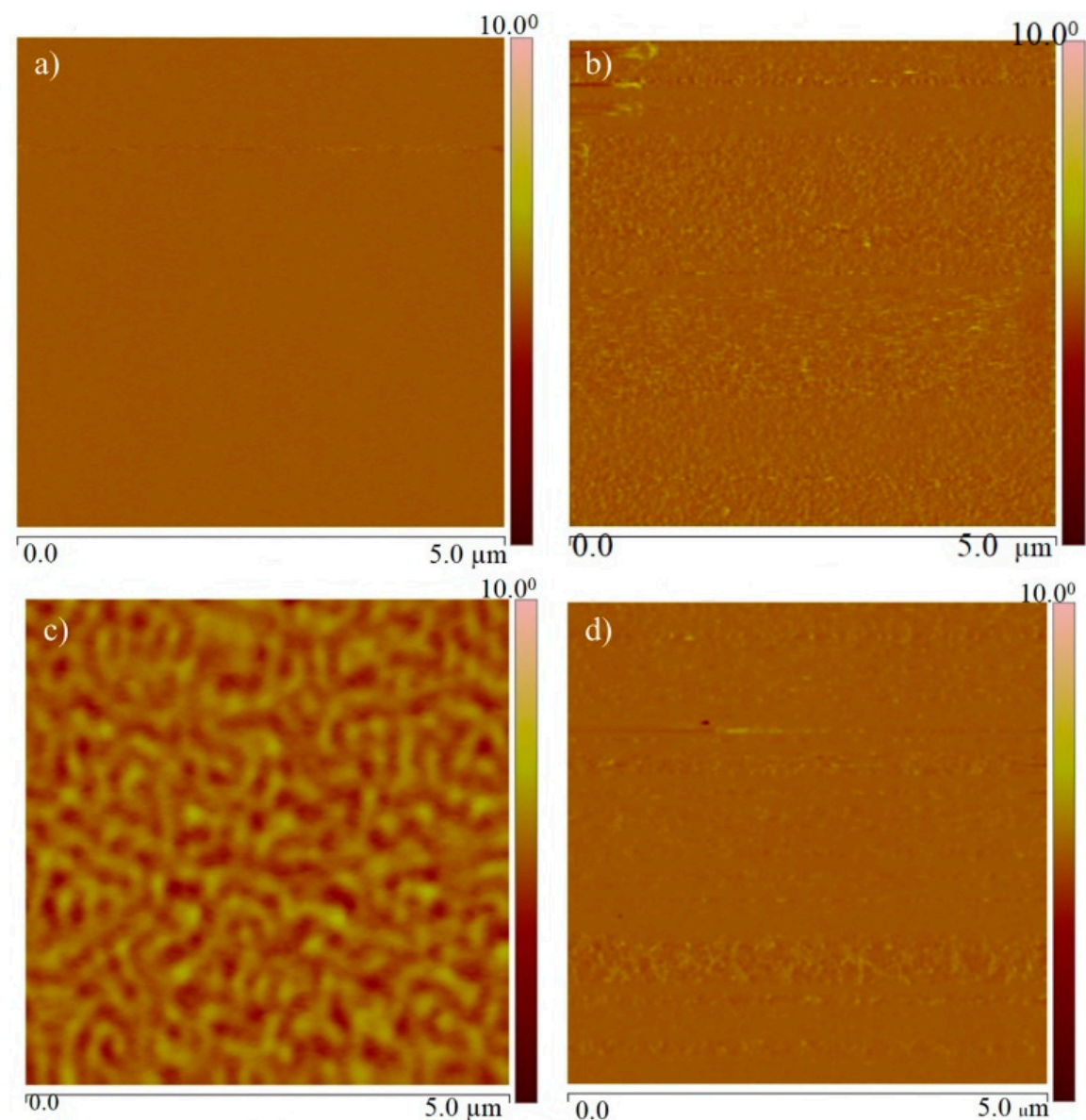

Figure 9. Magnetic force microscopy (MFM) images of (a) pure Ni film and films annealed at $400{ }^{\circ} \mathrm{C}$ for (b) $60 \mathrm{~min}$, (c) $90 \mathrm{~min}$, and (d) $120 \mathrm{~min}$. Reprinted with permission from [95], Copyright 2019 Elsevier.

Ravikumar et al. investigated thermal oxidation in $\mathrm{Ni}$ films of different thickness and found the relative amounts of $\mathrm{Ni}$ and $\mathrm{NiO}$ in films annealed at $400{ }^{\circ} \mathrm{C}$ to strongly depend on the thickness of the original Ni layer, while an annealing temperature of $500{ }^{\circ} \mathrm{C}$ resulted in full oxidation of the Ni film [96]. The exchange bias was found to depend on the $\mathrm{Ni}: \mathrm{NiO}$ 
ratio; it was largest for a sample of original thickness $200 \mathrm{~nm}$ and near zero for a sample of thickness $50 \mathrm{~nm}$ [96]. In another study, the same group found the largest EB in a sample annealed in vacuum at $500{ }^{\circ} \mathrm{C}[97]$.

To modify such $\mathrm{Ni} / \mathrm{NiO}$ thin film systems, Raghavan et al. used ion implantation with $\mathrm{Cu}$ ions which were deposited in the $\mathrm{NiO}$ layer and along the interface [98]. By this, an initial increase of the exchange bias, followed by a decrease with increasing ion flux was found. Besides, the changes in the exchange bias were larger when the $\mathrm{Cu}$ ions were implanted in the AFM, as compared to ion implantation along the interface between ferromagnet and antiferromagnet [98].

Another quite interesting finding was recently reported by Anyfantis et al., who showed that the anisotropy orientation of $\mathrm{Ni} / \mathrm{NiO}$ multilayers after mild annealing could be modified from in-plane to perpendicular, clearly showing out-of-plane domains at remanence [99]. The change of the effective uniaxial magnetic anisotropy constant was attributed to a significant change of the volume anisotropy, while the surface anisotropy showed a small decrease due to roughening during annealing.

Zhang et al. combined a $\mathrm{Ni} / \mathrm{NiO}(001)$ thin film system with a ferroelectric substrate which could be used to modify the ferroelectric strain on the AFM layer by an out-of-plane electric field [100]. In this way, they could switch off the exchange bias by an electric field cycle, which was attributed to switching of the AFM domains due to the piezoelectric strain in the $\mathrm{NiO}$ layer.

An interesting application of $\mathrm{Ni} / \mathrm{NiO}$ thin films was reported by You and Che [101]. They prepared plate-like $\mathrm{Ni} / \mathrm{NiO}$ composites with strong magnetic coupling and used them for microwave absorption, finding a strong reflection loss and a broad bandwidth.

As these examples show, $\mathrm{Ni} / \mathrm{NiO}$ also may show interesting effects, while it is less often investigated than $\mathrm{Co} / \mathrm{CoO}$ exchange bias systems. Nevertheless, both systems usually show relatively smooth hysteresis loops which may be asymmetric, but are normally not very unusual. This is different in the systems presented in the next sections.

\section{3. $\mathrm{Fe} / \mathrm{FeF} \mathrm{F}_{2}$ Thin Film Systems}

$\mathrm{Fe} / \mathrm{FeF}_{2}$ belongs to the systems which were regularly investigated around the year 2000, leading to interesting experimental findings and corresponding theoretical models $[2,5,50,102-106]$, finding, e.g., a perpendicular coupling $\left(90^{\circ}\right.$ coupling) between FM and AFM spins [103], a positive exchange bias [20,52] or asymmetric magnetization reversal processes in $\mathrm{Fe} / \mathrm{FeF}_{2}$ with twinned $\mathrm{AFM}$, i.e., domain wall propagation and motion or magnetization rotation, depending on the side of the hysteresis loop [104].

In spite of these interesting properties of this system, research on $\mathrm{Fe} / \mathrm{FeF}_{2}$ during recent years was only found for core/shell nanoparticles [107]. Regarding $\mathrm{Fe} / \mathrm{FeF}_{2}$ bilayers, Tangarife et al. combined experimental results with ab initio calculations of cohesive energies of different $\mathrm{Fe}$ and $\mathrm{FeF}_{2}$ phases, finding a reordering of the atomic positions of $\mathrm{Fe}$ and $\mathrm{F}$ atoms along the interface, resulting in an increase of the charge transfer between the atoms, giving a possible explanation for the exchange bias as originating from asymmetric electric charge transfer in different spin channels [108].

Other recent research of this interesting system could not be found in recent literature, although this system - especially due to the possibility to prepare the antiferromagnet in epitaxial, twinned or grain structure-surely still enables interesting new findings.

\section{4. $\mathrm{Fe} / \mathrm{MnF} 2$ Thin Film Systems}

Similar to $\mathrm{Fe} / \mathrm{FeF}_{2}, \mathrm{Fe} / \mathrm{MnF}_{2}$ systems have often been investigated around the year 2000 [7,8,49-51]. Both antiferromagnets show a strong anisotropy [51]; however, the interface exchange constant of $\mathrm{Fe} / \mathrm{FeF}_{2}$ is more than three times the value of $\mathrm{Fe} / \mathrm{MnF}_{2}$, while the stiffness parameter of $\mathrm{Fe} / \mathrm{FeF}_{2}$ is nearly one order of magnitude larger than the value for $\mathrm{Fe} / \mathrm{MnF}_{2}$ [51], suggesting differences in the exchange bias behavior. Indeed, in $\mathrm{Fe} / \mathrm{MnF}_{2}$ effects such as a sudden onset of a fourfold anisotropy in addition to the unidirectional exchange bias below the Néel temperature of $\mathrm{MnF}_{2}$ [109], an antiproportional 
correlation of coercive field and exchange bias with the Fe layer thickness [110] and an alignment of the magnetization with the cooling-field direction as an intermediate step during magnetization reversal [111] were found. Especially the nearly rectangular peaks in the transverse magnetization, correlated with pronounced steps in the longitudinal hysteresis loop, as depicted in Figure 10 [111], are characteristic for this system [8,111].

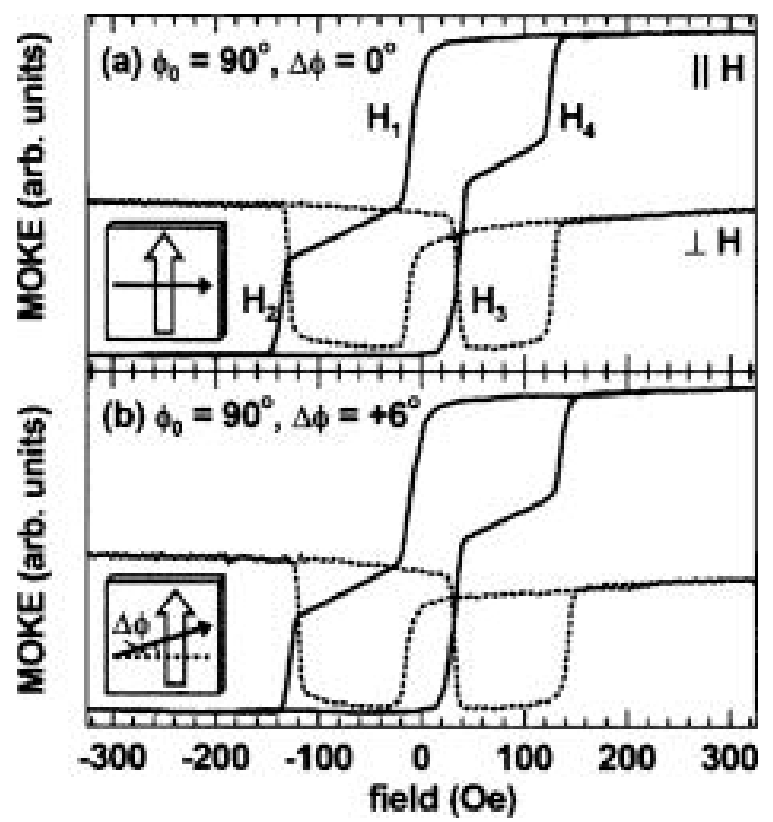

Figure 10. Magnetization reversal measured at low temperature at angles (a) perpendicular and (b) nearly perpendicular to the cooling field direction. Reprinted with permission from [111], Copyright 2005 AIP Publishing.

In spite of these highly interesting characteristics of this EB system which could, e.g., be used for quaternary storages devices [112], no studies from the last years were found in the literature.

\section{Thin Film Systems from Other Materials}

Besides these relatively simple systems which were used as the base for several exchange bias models, several other systems have been investigated during the last years. Here, we point out some interesting effects found in these material systems.

Shameem and Kumar, e.g., used magnetron sputtering to deposit polycrystalline $\mathrm{Fe}_{3} \mathrm{O}_{4}$ ferrimagnetic films [113]. Their granular single-phase $\mathrm{Fe}_{3} \mathrm{O}_{4}$ films revealed no other crystalline phases. Interestingly, in the grains a core-shell structure was built, with a ferrimagnetic core surrounded by spin-glass shell which resulted in an exchange bias at low temperatures. A strong athermal training effect was found (Figure 11), which may be extrapolated to an exchange bias value near zero for large numbers of hysteresis loops [113].

Another material in which an exchange bias can occur without a second layer is Fe-rich FeRh. Lee et al. prepared thin films by magnetron sputtering from an $\mathrm{Fe}_{67} \mathrm{Rh}_{33}$ target onto an $\mathrm{MgO}(001)$ substrate [114]. By annealing, the crystallinity of the films and also the exchange bias increased, including a significant increase of the EB from 1073 to $1173 \mathrm{~K}$ (Figure 12) [114]. This exchange bias was attributed to a strain-induced AFM/FM phase transition at the interface [115], in this way automatically forming an FM/AFM double layer system. 

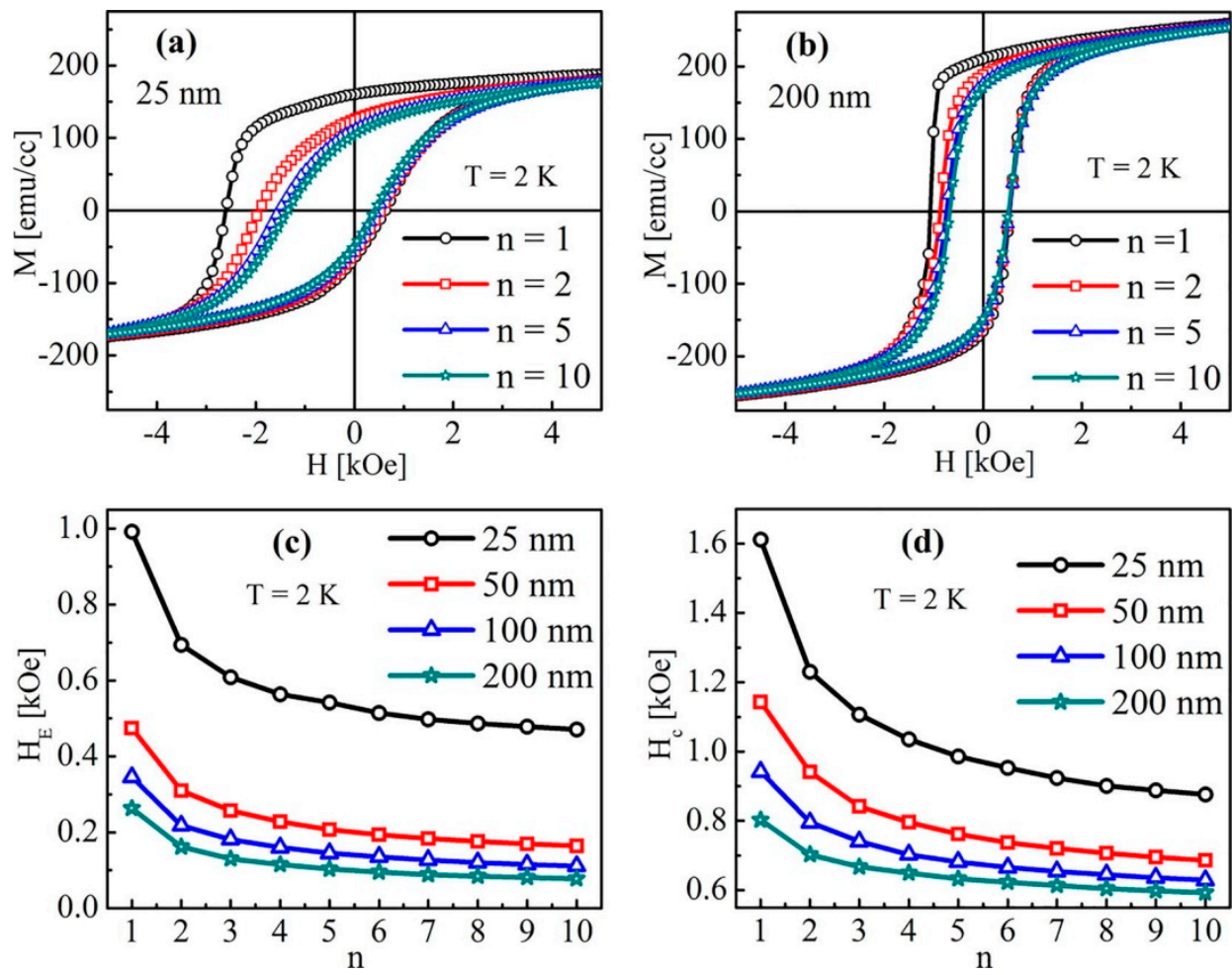

Figure 11. Hysteresis loops, measured at $T=2 \mathrm{~K}$ after field cooling for a $\mathrm{Fe}_{3} \mathrm{O}_{4}$ film thickness of (a) $25 \mathrm{~nm}$, (b) $200 \mathrm{~nm}$; dependence of (c) the exchange bias field HE, (d) the coercive field HC on the number of cycles. Reprinted with permission from [113], Copyright 2018 Elsevier.
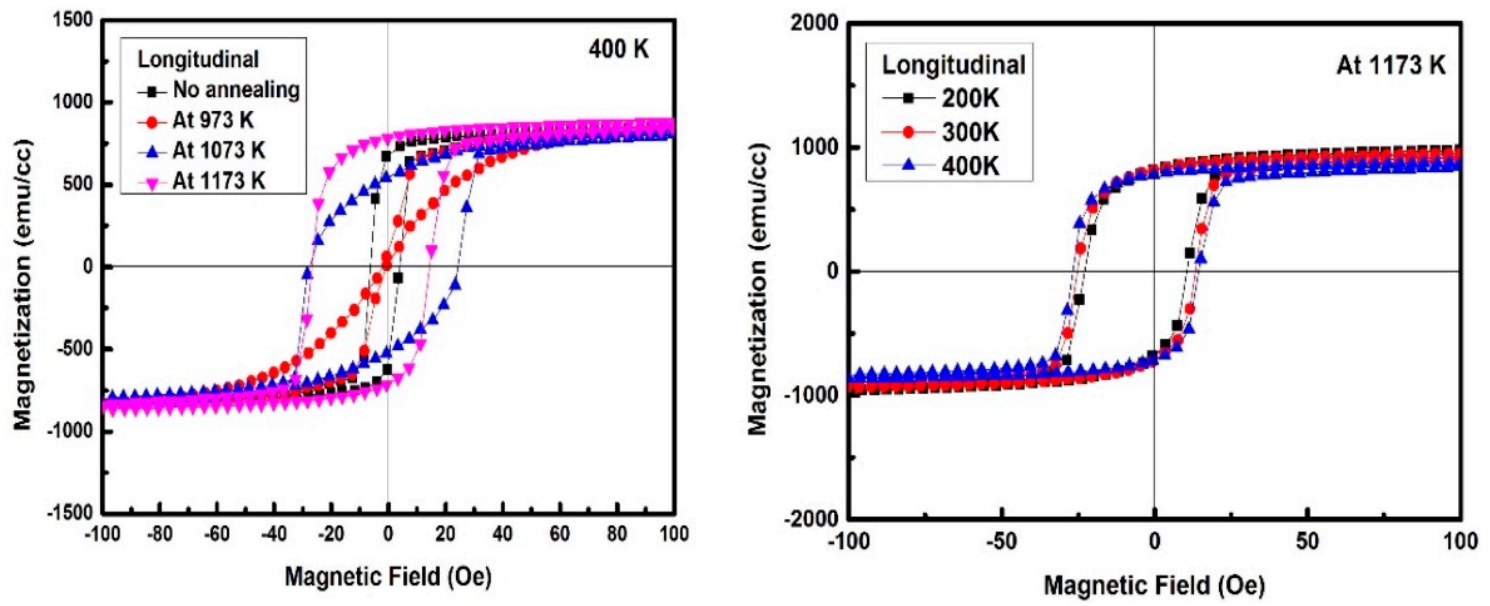

Figure 12. Hysteresis curves of a FeRh thin film, measured at $400 \mathrm{~K}$ after annealing at different temperatures (left panel) and measured at different temperatures after annealing at $1173 \mathrm{~K}$. Reprinted with permission from [114], Copyright 2019 Elsevier.

$\mathrm{MgFe}_{2} \mathrm{O}_{4}$ is another material which can exhibit an exchange bias in a single phase. As Mallick and Kumar pointed out, this effect occurred in $\mathrm{MgFe}_{2} \mathrm{O}_{4}(111)$ thin films on $\mathrm{MgO}$-buffered $\mathrm{Si}(100)$, but not in stoichiometric powder of the same material, suggesting that the EB is based on the growth-induced magnetic and structural disorder [116]. While here also a training effect was found, the temperature- and cooling field-dependence of coercivity and EB could not be explained as in conventional coupled FM/AFM systems, but by a unidirectional anisotropy along the field direction due to a disordered layer with random spin orientation along the grain boundaries which supported the AFM interaction between the ferrimagnetic grains [116]. 
Another material exhibiting an exchange bias in a single phase is the Heusler alloy Ni$\mathrm{Mn}-\mathrm{Sn}$, showing an austenitic phase at room temperature and a martensitic transformation at lower temperatures. The exchange bias in this material can be attributed to coexisting AFM and FM phases due to different occupations of the Mn atoms [117-119].

Finally, it should be mentioned that double-perovskites can also show single-phase exchange bias and even the so-called spontaneous exchange bias, i.e., an exchange bias oriented due to the first hysteresis loop at low temperatures [120,121]. While not yet prepared as thin films, this interesting material class may offer more surprising and technologically applicable effects related to the exchange bias.

In $\mathrm{La}_{0.7} \mathrm{Sr}_{0.3} \mathrm{MnO}_{3} / \mathrm{NiO}$ nanocomposites, Panchal et al. found a spontaneous exchange bias after zero-field cooling [122]. The interface between both components showed competing ferromagnetic and antiferromagnetic interaction, resulting in a spin-glass behavior. The spontaneous EB was attributed to ferromagnetic coupling of unstable AFM interface spins with the ferromagnetic phase.

Wu et al. found a transition of the EB in this system in the form of multilayer and columnar structures, grown on $\mathrm{SrTiO}_{3}$ substrates using pulsed laser deposition, from inplane to out-of-plane, depending on the microstructure [123]. This finding was explained by the interface strain-induced spin coupling reorientation dominating the orientation of the EB in this system, thus allowing for tailoring the EB orientation by the interface strain.

FeSn has an interesting crystalline structure as it is composed of subsequent $\mathrm{Fe}_{3} \mathrm{Sn}$ Kagome planes and Sn plane with honeycomb structure. Combining this AFM with ferromagnetic permalloy (Py), Khadka et al. found a large exchange bias with steep training effect [124].

Another quite interesting material is $\mathrm{BiFeO}_{3}$ which is a possible candidate for domain wall-based devices. As Chen et al. showed, introducing a dielectric layer (LBFO) between a $\mathrm{SrRuO}_{3}$ bottom electrode and the multiferroic $\mathrm{BiFeO}_{3}$ layer enables control of the domain patterns of $\mathrm{BiFeO}_{3}$ films [125]. Coupling this system with a ferromagnet, e.g., CoFe, results in an exchange bias which can be controlled by a magnetic field during layer growth (Figure 13).
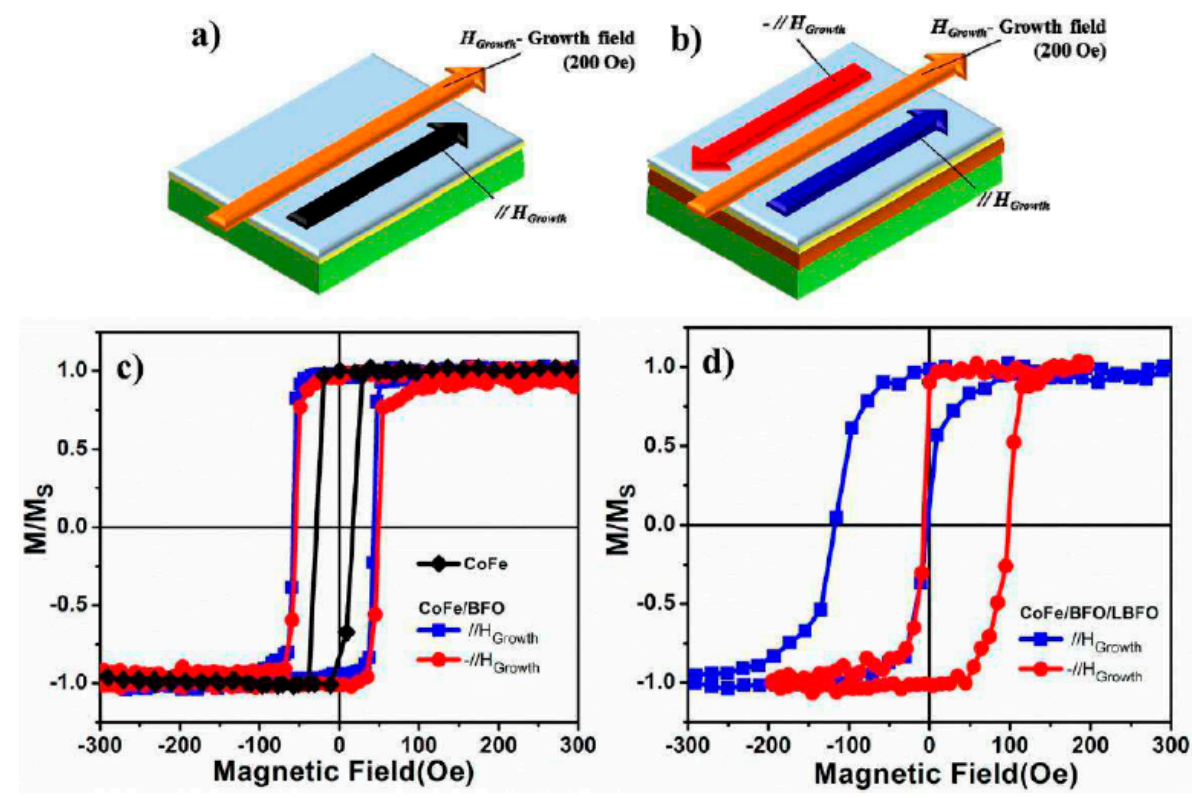

Figure 13. Applied magnetic fields during CoFe growth and measurement of (a) CoFe and (b) $\mathrm{CoFe} / \mathrm{BiFeO}_{3}$ with or without dielectric LBFO layer; hysteresis loops measured for systems (c) without and (d) with dielectric layer. Reprinted with permission from [125], Copyright 2017 American Chemical Society. 
As these few examples show, there are still many new exchange bias systems-or even single-phase exchange bias materials- to be discovered, and new phenomena can be expected to be found also in well-known systems. This section thus can only give a brief overview of some highly interesting findings from the last years. Such new single-phase materials or material systems are highly interesting not only due to understanding the exchange bias effect better in basic research, but also due to potential new applications. Controlling the magnetism in the antiferromagnetic semiconductor $\mathrm{CrI}_{3}$, e.g., by a small gate voltage in a field-effect device resulted in a large magneto-electric effect whose sign is defined by the interlayer AFM order and thus the interlayer exchange bias. This finding can be used in novel information technology devices [126].

The aforementioned voltage-control of the exchange bias [71] can be used for logic and memory techniques. Ferromagnetic films with perpendicular magnetization can be switched deterministically using the spin Hall effect, if an antiferromagnetic layer is added for symmetry breaking by an in-plane exchange bias field [127]. In a more complicated multi-layer memristive device based on this principle, Borders et al. showed even an analogue-like behavior, enabling pattern recognition by a simple associative memory system [128].

Surface acoustic wave sensors were produced using Co/IrMn multilayers [129]. Polewczyk et al. prepared high magnetic field sensors with reduced response hysteresis of the ferromagnet by carefully modeling the magnetic properties of the system and tailoring the magneto-acoustic response by modifying sensor shape and material properties.

Generally, for spintronic and magnetic storage devices as well as sensors it is useful to prepare systems with room temperature exchange bias. This is not given in $\mathrm{Fe} / \mathrm{MnF}_{2}$, $\mathrm{FeFeF}_{2}$ and many other traditional systems, which stimulates searching for new ferromagnetic/antiferromagnetic systems or even single-phase exchange bias materials for such future applications [130].

\section{Conclusions}

While the exchange bias effect is now known for more than 60 years, there are still regularly new, surprising phenomena found, even in apparently well-known systems, and new materials which exhibit exchange bias under unexpected conditions. Some of them are the spontaneous exchange bias, oriented due to the first hysteresis loop at low temperatures, an exchange bias in systems not consisting of the typical AFM/FM combinations, and single-phase materials exhibiting exchange bias. Especially systems exhibiting an exchange bias above room temperature are of technological interest for spintronics and memory devices.

Future research should, however, not only concentrate on these technologically promising material systems, but also develop new models to understand the exchange bias effect in all their manifestations better, and investigate new systems and effects to enable a deeper knowledge of exchange bias systems.

We hope that this brief review of theoretical exchange bias models and recent experimental findings will stimulate the reader to investigate the here mentioned and other materials and possibly detect again new exchange bias materials and phenomena.

Author Contributions: Conceptualization, T.B. and A.E.; writing-review and editing, A.E. and T.B.; visualization, A.E. All authors have read and agreed to the published version of the manuscript.

Funding: The APC is funded by the Open Access Publication Fund of Bielefeld University of Applied Sciences.

Institutional Review Board Statement: Not applicable.

Informed Consent Statement: Not applicable.

Data Availability Statement: No new data were created or analyzed in this study. Data sharing is not applicable to this article. 
Conflicts of Interest: The authors declare no conflict of interest. The funders had no role in the design of the study; in the collection, analyses, or interpretation of data; in the writing of the manuscript; or in the decision to publish the results.

\section{References}

1. Meiklejohn, W.H.; Bean, C.P. New magnetic anisotropy. Phys. Rev. 1956, 102, 1413-1414. [CrossRef]

2. Nogués, J.; Moran, T.J.; Lederman, D.; Schuller, I.K.; Rao, K.V. Role of interfacial structure on exchange-biased FeF 2 -Fe. Phys. Rev. B 1999, 59, 6984-6993. [CrossRef]

3. Blachowicz, T.; Tillmanns, A.; Fraune, M.; Beschoten, B.; Güntherodt, G. Exchange-bias in (110)-oriented CoO/Co bilayers with different magnetocrystalline anisotropies. Phys. Rev. B 2007, 75, 054425. [CrossRef]

4. Schneider, V.; Reinholdt, A.; Kreibig, U.; Weirich, T.; Güntherodt, G.; Beschoten, B.; Tillmanns, A.; Krenn, H.; Rumpf, K.; Granitzer, P. Structural and Magnetic Properties of Ni/NiOxide and Co/CoOxide Core/Shell Nanoparticles and their possible Use for Ferrofluids. Z. Phys. Chem. 2006, 220, 173-187. [CrossRef]

5. Tillmanns, A.; Oertker, S.; Beschoten, B.; Güntherodt, G.; Eisenmenger, J.; Schuller, I.K. Angular dependence and origin of asymmetric magnetization reversal in exchange-biased Fe/FeF 2 (110). Phys. Rev. B 2008, 78, 012401. [CrossRef]

6. Eisenmenger, J.; Li, Z.-P.; Macedo, W.A.A.; Schuller, I.K. Exchange bias and asymmetric reversal in nanostructures dot arrays. Phys. Rev. Lett. 2005, 94, 057203. [CrossRef] [PubMed]

7. Macedo, W.A.A.; Sahoo, B.; Kuncser, V.; Eisenmenger, J.; Felner, I.; Nogués, J.; Liu, K.; Keune, W.; Schuller, I.K. Changes in ferromagnetic spin structure induced by exchange bias in $\mathrm{Fe} / \mathrm{MnF}_{2}$ films. Phys. Rev. B 2004, 70, 224414. [CrossRef]

8. Tillmanns, A.; Oertker, S.; Beschoten, B.; Güntherodt, G.; Leighton, C.; Schuller, I.K.; Nogués, J. Magneto-optical study of magnetization reversal asymmetry in exchange bias. Appl. Phys. Lett. 2006, 89, 202512. [CrossRef]

9. Hussain, Z.; Bera, A.K.; Dev, A.S.; Kumar, D.; Reddy, V.R. Exchange bias effect in $\mathrm{Fe} / \mathrm{LaAlO}_{3}$ : An interface induced effect. J. Alloys Comp. 2020, 849, 156484. [CrossRef]

10. Zhang, B.M. Correlation of microstructure with magnetic properties in $\operatorname{Pr}_{0.67} \mathrm{Sr}_{0.33} \mathrm{MnO}_{3}$ thin films. J. Mater. Sci. Mater. Electron. 2020, 31, 19875-19882. [CrossRef]

11. Nogués, J.; Sort, J.; Langlais, V.; Skumryev, V.; Surinach, S.; Munoz, J.S.; Baró, M.D. Exchange bias in nanostructures. Phys. Rep. 2005, 422, 65-117. [CrossRef]

12. Laureti, S.; Suck, S.Y.; Haas, H.; Prestat, E.; Bourgeouis, O.; Givord, D. Size dependence of exchange bias in Co/CoO nanostructures. Phys. Rev. Lett. 2012, 108, 077205. [CrossRef] [PubMed]

13. Javed, K.; Li, W.J.; Ali, S.S.; Shi, D.W.; Khan, U.; Riaz, S.; Han, X.F. Enhanced exchange bias and improved ferromagnetic properties in Permalloy-BiFe ${ }_{0.95} \mathrm{Co}_{0.05} \mathrm{O}_{3}$ core-shell nanostructures. Sci. Rep. 2016, 5, 18203. [CrossRef] [PubMed]

14. Barreto, P.G.; Sousa, M.A.; Pelegrini, F.; Alayo, W.; Litterst, F.J.; Baggio-Saitovitch, E. Ferromagnetic resonance study of the misalignment between anisotropy axes in exchange-biased NiFe/FeMn/Co trilayers. Appl. Phys. Lett. 2014, 104, 202403. [CrossRef]

15. Sing, S.; Kumar, P.; Gupta, A.; Kumar, D. In-situ study of exchange-bias in interlayer coupled Co/CoO/Co trilayer structure. J Magn. Magn. Mater. 2020, 513, 167186. [CrossRef]

16. Nogués, J.; Schuller, I.K. Exchange bias. J. Magn. Magn. Mat. 1999, 192, 203. [CrossRef]

17. Thomas, S.; Reethu, K.; Thanveer, T.; Myint, M.T.Z.; Al-Harthi, S.H. Effect of shell thickness on the exchange bias blocking temperature and coercivity in Co-CoO core-shell nanoparticles. J. Appl. Phys. 2017, 122, 063902. [CrossRef]

18. Ali, M.; Adie, P.; Marrows, C.H.; Greig, D.; Hickey, B.J.; Stamps, R.L. Exchange bias using a spin glass. Nat. Mater. 2007, 6, 70-75. [CrossRef]

19. Moran, T.J.; Schuller, I.K. Effects of cooling field strength on exchange anisotropy at permalloy/CoO interfaces. J. Appl. Phys. 1996, 79, 5109. [CrossRef]

20. Nogués, J.; Lederman, D.; Moran, T.J.; Schuller, I.K. Positive exchange bias in Fe-Fe 2 bilayers. Phys. Rev. Lett. 1996, 76, 4624. [CrossRef]

21. Nogués, J.; Leighton, C.; Schuller, I.K. Correlation between antiferromagnetic interface coupling and positive exchange bias. Phys. Rev. B 2000, 61, 1315. [CrossRef]

22. Ambrose, T.; Chien, C.L. Dependence of exchange field and coercitivity on cooling field in NiFe/CoO bilayers. J. Appl. Phys. 1998, 83, 7222. [CrossRef]

23. Leighton, C.; Nogués, J.; Suhl, H.; Schuller, I.K. Competing interfacial exchange and Zeeman energies in exchange biases bilayers. Phys. Rev. B 1999, 60, 12837. [CrossRef]

24. Hwang, D.G.; Lee, S.S.; Park, C.M. Effect of roughness slope on exchange biasing in NiO spinvalves. Appl. Phys. Lett. 1998, 72, 2162. [CrossRef]

25. Moran, T.J.; Gallego, J.M.; Schuller, I.K. Increased exchange anisotropy due to disorder at permalloy/CoO interfaces. J. Appl. Phys. 1995, 78, 1887. [CrossRef]

26. Polisetty, S.; Sahoo, S.; Binek, C. Scaling behavior of the exchange-bias training effect. Phys. Rev. B 2007, 76, 184423. [CrossRef]

27. Qiu, X.P.; Yang, D.Z.; Zhou, S.M.; Chantrell, R.; O'Grady, K.; Nowak, U.; Du, J.; Bai, X.J.; Sun, L. Rotation of the pinning direction in the exchange bias training effect in polycrystalline NiFe/FeMn bilayers. Phys. Rev. Lett. 2008, 101, 147207. [CrossRef] 
28. Brems, S.; Temst, K.; van Haesendonk, C. Origin of the training effect and asymmetry of the magnetization in polycrystalline exchange bias systems. Phys. Rev. Lett. 2007, 99, 067201. [CrossRef]

29. Binek, C.; Polisetty, S.; He, X.; Berger, A. Exchange bias training effect in coupled all ferromagnetic bilayer structures. Phys. Rev. Lett. 2006, 96, 067201. [CrossRef]

30. Brück, S.; Schütz, G.; Goering, E.; Ji, X.S.; Krishnan, K.M. Uncompensated moments in the MnPd/Fe exchange bias system. Phys. Rev. Lett. 2008, 101, 126402. [CrossRef]

31. Meiklejohn, W.H. Exchange anisotropy-A review. J. Appl. Phys. 1962, 33, 1328-1335. [CrossRef]

32. Néel, L. Étude théorique du couplage ferro-antiferromagnétique dans les couches minces. Ann. Phys. 1967, 14, 61-80. [CrossRef]

33. Kiwi, M. Exchange bias theory. J. Magn. Magn. Mater. 2001, 234, 584-595. [CrossRef]

34. Malozemoff, A.P. Random-field model of exchange bias anisotropy at rough ferromagnetic-antiferromagnetic interfaces. Phys. Rev. B 1987, 35, 3679. [CrossRef]

35. Malozemoff, A.P. Mechanisms of exchange anisotropy. J. Appl. Phys. 1988, 63, 3874-3879. [CrossRef]

36. Malozemoff, A.P. Heisenberg to Ising crossover in a random-field model with uniaxial anisotropy. Phys. Rev. B 1998, $37,7673$. [CrossRef] [PubMed]

37. Mauri, D.; Siegmann, H.C.; Bagus, P.S.; Kay, E. Simple model for thin ferromagnetic films exchange coupled to an antiferromagnetic substrate. J. Appl. Phys. 1987, 62, 3047-3049. [CrossRef]

38. Meiklejohn, W.H.; Bean, C.P. New magnetic anisotropy. Phys. Rev. 1957, 105, 904. [CrossRef]

39. Schulthess, T.C.; Butler, W.H. Consequences of spin-flop coupling in exchange biased films. Phys. Rev. Lett. 1998, 81, 4516. [CrossRef]

40. Schulthess, T.C.; Butler, W.H. Coupling mechanisms in exchange biased films. J. Appl. Phys. 1999, 85, 5510. [CrossRef]

41. Takano, K.; Kodama, R.H.; Berkowitz, A.E.; Cao, W.; Thomas, G. Interfacial uncompensated antiferromagnetic spins: Role in unidirectional anisotropy in polycrystalline $\mathrm{Ni}_{81} \mathrm{~F}_{19} / \mathrm{CoO}$ bilayers. Phys. Rev. Lett. 1997, 79, 1130. [CrossRef]

42. Takano, K.; Kodama, R.H.; Berkowitz, A.E.; Cao, W.; Thomas, G. Role of interfacial uncompensated antiferromagnetic spins in unidirectional anisotropy in $\mathrm{Ni}_{81} \mathrm{~F}_{19} / \mathrm{CoO}$ bilayers. J. Appl. Phys. 1998, 83, 6888. [CrossRef]

43. Stiles, M.D.; McMichael, R.D. Model for exchange bias in polycrystalline ferromagnet-antiferromagnet bilayers. Phys. Rev. B 1999, 59, 3722. [CrossRef]

44. Miltényi, P.; Gierlings, M.; Keller, M.; Beschoten, B.; Güntherodt, G.; Nowak, U.; Usadel, K.D. Diluted antiferromagnets in exchange bias: Proof of the domain state model. Phys. Rev. Lett. 2000, 84, 4224. [CrossRef] [PubMed]

45. Nowak, U.; Usadel, K.D.; Keller, J.; Miltényi, P.; Beschoten, B.; Güntherodt, G. Domain state model for exchange bias. I. Theory. Phys. Rev. B 2002, 66, 014430. [CrossRef]

46. Keller, J.; Miltényi, P.; Beschoten, B.; Güntherodt, G.; Nowak, U.; Usadel, K.D. Domain state model for exchange bias. II. Experiments. Phys. Rev. B 2002, 66, 014431. [CrossRef]

47. Beschoten, B.; Keller, J.; Tillmanns, A.; Miltényi, P.; Güntherodt, G. Domain state model for exchange bias: Training effect of diluted $\mathrm{Co}_{1-y} \mathrm{O}$ on exchange bias in Co/CoO. IEEE Trans. Magn. 2002, 38, 2744. [CrossRef]

48. Nowak, U.; Misra, A.; Usadel, K.D. Modeling exchange bias microscopically. J. Magn. Magn. Mater. 2002, 240, 243-247. [CrossRef]

49. Nowak, U.; Misra, A.; Usadel, K.D. Domain staate model for exchange bias. J. Appl. Phys. 2001, 89, 7269-7271. [CrossRef]

50. Kiwi, M.; Mejía-López, J.; Portugal, R.D.; Ramírez, R. Exchange bias model for Fe/FeF 2 : Role of domains in the ferromagnet. Europhys. Lett. 1999, 48, 573-579. [CrossRef]

51. Kiwi, M.; Mejía-López, J.; Portugal, R.D.; Ramírez, R. Exchange-bias systems with compensated interfaces. Appl. Phys. Lett. 1999, 75, 3995-3997. [CrossRef]

52. Kiwi, M.; Mejía-López, J.; Portugal, R.D.; Ramírez, R. Positive exchange bias model: Fe/FeF 2 and Fe/MnF 2 bilayers. Solid State Comm. 2000, 116, 315-319. [CrossRef]

53. Nolting, F.; Scholl, A.; Stöhr, J.; Seo, J.W.; Fompeyrine, J.; Siegwart, H.; Locquet, J.-P.; Anders, S.; Lüning, J.; Fullerton, E.E.; et al. Direct observation of the alignment of ferromagnetic spins by antiferromagnetic spins. Nature 2000, 405, 767-769. [CrossRef] [PubMed]

54. Matsuyama, H.; Haginoya, C.; Koike, K. Microscopic imaging of Fe magnetic domains exchange coupled with those in a NiO(001) surface. Phys. Rev. Lett. 2000, 85, 646-649. [CrossRef] [PubMed]

55. Radu, F.; Westphalen, A.; Theis-Bröhl, K.; Zabel, H. Quantitative description of the azimuthal dependence of the exchange bias effect. J. Phys. Condens. Matter 2006, 18, L29-L36. [CrossRef]

56. Harres, A.; Geshev, J. A polycrystalline model for magnetic exchange bias. J. Phys. Condens. Matter 2012, 24, 326004. [CrossRef] [PubMed]

57. Saha, J.; Victora, R.H. Large scale micromagnetic simulation for the exchange interaction between a polycrystalline antiferromagnet and a ferromagnet. Phys. Rev. B 2006, 73, 104433. [CrossRef]

58. Choo, D.; Chantrell, R.W.; Lamberton, R.; Johnston, A.; O'Grady, K. A model of the magnetic properties of coupled ferromagnetic/antiferromagnetic bilayers. J. Appl. Phys. 2007, 101, 09E521. [CrossRef]

59. O'Grady, K.; Fernandez-Outon, L.E.; Vallejo-Fernandez, G. A new paradigm for exchange bias in polycrystalline thin films. J. Magn. Magn. Mater. 2010, 322, 883-899. [CrossRef]

60. Mangin, S.; Montaigne, F.; Schuhl, A. Interface domain wall and exchange bias phenomena in ferrimagnetic/ferrimagnetic bilayers. Phys. Rev. B 2003, 68, 140404. [CrossRef] 
61. Fullerton, E.E.; Jiang, J.S.; Grimsditch, M.; Sowers, C.H.; Bader, S.D. Exchange-spring behavior in epitaxial hard/soft magnetic bilayers. Phys. Rev. B 1998, 58, 12193. [CrossRef]

62. Montaigne, F.; Mangin, S.; Henry, Y. Transitions of magnetic configuration at the interface of exchange-coupled bilayers: $\mathrm{TbFe} / \mathrm{GdFe}$ as a model system. Phys. Rev. B 2003, 67, 144412. [CrossRef]

63. Stamps, R.L. Mechanism of exchange bias: Partial wall pinning, and fluctuations. J. Magn. Magn. Mater. 2002, 242-245, 139-145. [CrossRef]

64. Morales, R.; Basaran, A.C.; Villegas, J.E.; Navas, D.; Soriano, N.; Mora, B.; Redondo, C.; Batlle, X.; Schuller, I.K. Exchange-bias phenomenon: The role of the ferromagnetic spin structure. Phys. Rev. Lett. 2015, 114, 097202. [CrossRef] [PubMed]

65. Dimitriadis, V.; Kechrakos, D.; Chubykalo-Fesenko, O.; Tsiantos, V. Shape-dependent exchange bias effect in magnetic nanoparticles with core-shell morphology. Phys. Rev. B 2015, 92, 064420. [CrossRef]

66. Iglesias, Ò.; Labarta, A.; Batlle, X. Exchange bias phenomenology and models of core/shell nanoparticles. J. Nanosci. Nanotechnol. 2008, 8, 2761-2780. [CrossRef]

67. Simon, E.; Yanes, R.; Khmelevskyi, S.; Palotás, K.; Szunyogh, L.; Nowak, U. Magnetism and exchange-bias effect at the MnN/Fe interface. Phys. Rev. B 2018, 98, 094415. [CrossRef]

68. Khan, R.A.; Nembach, H.T.; Ali, M.; Shaw, J.M.; Marrows, C.H.; Moore, T.A. Magnetic domain texture and the DzyaloshinskiiMoriya interaction in $\mathrm{Pt} / \mathrm{Co} / \mathrm{IrMn}$ and $\mathrm{Pt} / \mathrm{Co} / \mathrm{FeMn}$ thin films with perpendicular exchange bias. Phys. Rev. B 2018, $98,064413$. [CrossRef]

69. Kolesnikov, A.G.; Stebliy, M.E.; Davydenko, A.V.; Kozlov, A.G.; Osmushko, I.S.; Korochentsev, V.V.; Ognev, A.V.; Gerasimenko, A.V.; Sadovnikov, A.V.; Gubanov, V.A.; et al. Magnetic properties and the interfacial Dzyaloshinskii-Moriya interaction in exchange biased $\mathrm{Pt} / \mathrm{Co} / \mathrm{Ni}_{x} \mathrm{O}_{y}$ films. Appl. Surf. Sci. 2021, 543, 148720. [CrossRef]

70. Fita, I.; Markovich, V.; Moskvin, A.S.; Wisniewski, A.; Puzniak, R.; Iwanowski, P.; Martin, C.; Maignan, A.; Carbonio, R.E.; Gutowska, M.U.; et al. Reversed exchange-bias effect associated with magnetism reversal in the weak ferrimagnet $\mathrm{LuFe}_{0.5} \mathrm{Cr}_{0.5} \mathrm{O}_{3}$. Phys. Rev. B 2018, 97, 104416. [CrossRef]

71. Manipatruni, S.; Nikonov, D.E.; Lin, C.-C.; Prasad, B.; Huang, Y.-L.; Damodaran, A.R.; Chen, Z.H.; Ramesh, R.; Young, I.A. Voltage control of unidirectional anisotropy in ferromagnet-multiferroic system. Sci. Adv. 2018, 4, eaat4229. [CrossRef]

72. Albisetti, E.; Petti, D.; Sala, G.; Silvani, R.; Tacchi, S.; Finizio, S.; Wintz, S.; Calò, A.; Zheng, X.R.; Raabe, J.; et al. Nanoscale spin-wave circuits based on engineered reconfigurable spin-textures. Comm. Phys. 2018, 1, 56. [CrossRef]

73. González, J.A.; Andrés, J.P.; López Antón, R.; de Toro, J.A.; Normile, P.S.; Muniz, P.; Riveiro, J.M.; Nogués, J. Maximizing exchange bias in $\mathrm{Co} / \mathrm{CoO}$ core/shell nanoparticles by lattice matching between the shell and the embedding matrix. Chem. Mater. 2017, 29, 5200-5206. [CrossRef]

74. Xing, Q.X.; Han, Z.L.; Zhao, S.F. Exchange bias of nanostructured films assembled with Co/CoO core-shell clusters. Mater. Lett. 2017, 188, 103-106. [CrossRef]

75. Guo, B.B.; Xu, Y.L.; Zhou, S.M. Morphology dependence of low temperatures exchange bias Co/CoO core-shell nanoparticles/spheres by eco-friendly solvothermal route. AIP Adv. 2018, 8, 115115. [CrossRef]

76. Salazar-Alvarez, G.; Geshev, J.; Agramunt-Puig, S.; Navau, C.; Sanchez, A.; Sort, J.; Nogués, J. Tunable high-field magnetization in strongly exchange-coupled freestanding $\mathrm{Co} / \mathrm{CoO}$ core/shell coaxial nanowires. ACS Appl. Mater. Interfaces 2016, 8, 22477-22483. [CrossRef] [PubMed]

77. Gandha, K.; Chaudhary, R.P.; Mohapatra, J.; Koymen, A.R.; Liu, J.P. Giant exchange bias and its angular dependence in Co/CoO core-shell nanowire assemblies. Phys. Lett. A 2017, 381, 2092-2096. [CrossRef]

78. Morales, R.; Flores, A.N.; Vargas, N.M.; Giuliani, J.; Schuller, I.K.; Monton, C. Ultradense arrays of sub-100 nm Co/CoO nanodisks for spintronics applications. ACS Appl. Nano Mater. 2020, 3, 4037-4044. [CrossRef]

79. Sharma, A.; Tripathi, J.; Tripathi, S.; Kumar, Y.; Ugochukwu, K.C.; Kumar, D.; Gupta, M.; Chaudhary, R.J. Exchange bias in $\mathrm{Co} / \mathrm{CoO}$ thin films deposited onto self-assembled nanosphere arrays. J. Magn. Magn. Mater. 2020, 510, 166599. [CrossRef]

80. López Antón, R.; González, J.A.; Andrés, J.P.; Normile, P.; Canales-Vázquez, J.; Muniz, P.; Riveiro, J.M.; de Toro, J.A. Exchange Bias Optimization by Controlled Oxidation of Cobalt Nanoparticle Films Prepared by Sputter Gas Aggregation. Nanomaterials 2017, 7, 61. [CrossRef]

81. Ehrmann, A.; Blachowicz, T. Angle and rotational direction dependent horizontal loop shift in epitaxial Co/CoO bilayers on $\mathrm{MgO}(100)$. AIP Adv. 2017, 7, 115223. [CrossRef]

82. Ehrmann, A.; Blachowicz, T. Asymmetric hysteresis loops in Co thin films. Condens. Matter 2020, 5, 71. [CrossRef]

83. Jochum, J.K.; Saerbeck, T.; Lazenka, V.; Joly, V.; Shan, S.C.; Boyen, H.-G.; Ternst, K.; Vantomme, A.; van Bael, M.J. Magnetic characterization of oblique angle deposited $\mathrm{Co} / \mathrm{CoO}$ on gold nanoparticles. J. Magn. Magn. Mater. 2019, 483, 76-82. [CrossRef]

84. Ovejero, J.G.; Godinho, V.; Lacroix, B.; García, M.A.; Hernando, A.; Fernández, A. Exchange bias and two steps magnetization reversal in porous Co/CoO layer. Mater. Des. 2019, 171, 107691. [CrossRef]

85. Hussain, Z.; Reddy, V.R. Kerr microscopy study of thermal and athermal training effects in a C/CoO exchange bias system. J. Appl. Phys. 2017, 122, 103903. [CrossRef]

86. Hussain, Z.; Reddy, V.R. Role of anti-ferromagnetic layer thickness in exchange bias: Case study of Co/CoO with Kerr microscopy. Mater. Res. Expr. 2019, 6, 066109. [CrossRef]

87. Kumar, D.; Singh, S.; Gupta, A. Effect of interface roughness on exchange coupling in polycrystalline Co/CoO bilayer structure: An in-situ investigation. J. Appl. Phys. 2016, 120, 085307. [CrossRef] 
88. Dobrynin, A.N.; Warin, P.; Vorobiev, A.; Givord, D. On the origin of positive exchange bias and coercivity enhancement in proximity to the blocking temperature. J. Magn. Magn. Mater. 2021, 520, 166707. [CrossRef]

89. Menéndez, E.; Silva, L.E.S.; Johann, G.; Sort, J.; Dias, T. Unraveling the origin of training in granular Co-CoO exchange bias systems with buried antiferromagnetic constituents. J. Magn. Magn. Mater. 2019, 478, 170-174. [CrossRef]

90. Roy, A.; de Toro, J.A.; Amaral, V.S.; Muniz, P.; Riveiro, J.M.; Ferreira, J.M.F. Exchange bias beyond the superparamagnetic blocking temperature of the antiferromagnet in a Ni-NiO nanoparticulate system. J. Appl. Phys. 2015, 115, 073904. [CrossRef]

91. Chaghouri, H.L.; Tuna, F.; Santhosh, P.N.; Thomas, P.J. Tiny Ni-NiO nanocrystals with exchange bias induced room temperature ferromagnetism. Solid State Comm. 2016, 230, 11-15. [CrossRef]

92. Yu, T.; Zhang, Z.W.; Xu, Y.H.; Liu, Y.; Li, W.J.; Nie, Y.; Zhang, X.; Xiang, G. Exchange bias coupling in NiO/Ni bilayer tubular nanostructures synthetized by electrodeposition and thermal oxidation. J. Magn. Magn. Mater. 2017, 429, 74-78. [CrossRef]

93. Amemiya, K.; Sakamaki, M. Effect of electric field on magnetism of Ni thin films via antiferromagnetic NiO. E J. Surf. Sci. Nanotech. 2018, 16, 186-189. [CrossRef]

94. De los Santos Valladares, L.; Ionescu, A.; Holmes, S.; Barnes, C.H.W.; Bustamante Dominguez, A.; Avalos Quispe, O.; Gonzalez, J.C.; Milana, S.; Barbone, M.; Ferrari, A.C.; et al. Characterization of Ni thin films following thermal oxidation in air. J. Vac. Sci. Technol. B 2014, 32, 051808. [CrossRef]

95. Raghavan, L.; Ojha, S.; Sulania, I.; Mishra, N.C.; Ranjith, K.M.; Baenitz, M.; Kanjilal, D. Thermal annealing induced competition of oxidation and grain growth in nickel thin films. Thin Solid Films 2019, 680, 40-47. [CrossRef]

96. Ravikumar, P.; Taparia, D.; Alagarsamy, P. Thickness-dependent thermal oxidation of Ni into NiO thin films. J. Supercond. Novel Magn. 2018, 31, 3761-3775. [CrossRef]

97. Ravikumar, P.; Kisan, B.; Perumal, A. Thickness dependent ferromagnetism in thermally decomposed NiO thin films. J. Magn. Magn. Mater. 2016, 418, 86-91. [CrossRef]

98. Raghavan, L.; Ranjith, K.M.; Baenitz, M.; Kanjilal, D. Control of exchange bias by modifying the antiferromagnet and ferromagnetantiferromagnet interface. Europhys. Lett. 2020, 130, 67006. [CrossRef]

99. Anyfantis, D.I.; Sarigiannidou, E.; Rapenne, L.; Stamatelatos, A.; Ntemogiannis, D.; Kapaklis, V.; Poulopoulos, P. Unexpected development of perpendicular magnetic anisotropy in $\mathrm{Ni} / \mathrm{NiO}$ multilayers after mild thermal annealing. IEEE Magn. Lett. 2019, 10, 6104105. [CrossRef]

100. Zhang, Y.-J.; Chen, J.-H.; Li, L.-L.; Ma, J.; Nan, C.-W.; Lin, Y.-H. Ferroelectric strain modulation of antiferromagnetic moments in $\mathrm{Ni} / \mathrm{NiO}$ ferromagnet/antiferromagnet heterostructures. Phys. Rev. B 2017, 95, 174420. [CrossRef]

101. You, W.B.; Che, R.C. Excellent NiO-Ni nanoplate microwave absorber via pinning effect of antiferromagnetic-ferromagnetic interface. ACS Appl. Mater. Interfaces 2018, 10, 15104-15111. [CrossRef] [PubMed]

102. Fitzsimmons, M.R.; Leighton, C.; Nogués, J.; Hoffmann, A.; Liu, K.; Majkrzak, C.K.; Dura, J.A.; Groves, J.R.; Springer, R.W.; Arendt, P.N.; et al. Influence of in-plane crystalline quality of an antiferromagnet on perpendicular exchange coupling and exchange bias. Phys. Rev. B 2002, 65, 134436. [CrossRef]

103. Moran, T.J.; Nogués, J.; Lederman, D.; Schuller, I.K. Perpendicular coupling at Fe-FeF 2 interfaces. Appl. Phys. Lett. 1998, $72,617$. [CrossRef]

104. Fitzsimmons, M.R.; Yashar, P.; Leighton, C.; Schuller, I.K.; Majkrzak, C.F.; Dura, J.A. Asymmetric magnetization reversal in exchange-biased hysteresis loops. Phys. Rev. Lett. 2000, 84, 3986. [CrossRef] [PubMed]

105. Dahlberg, E.D.; Miller, B.; Hill, B.; Jonsson, B.J.; Strom, V.; Rao, K.V.; Nogués, J.; Schuller, I.K. Measurements of the ferromagnetic/antiferromagnetic interfacial exchange energy in $\mathrm{CO} / \mathrm{CoO}$ and $\mathrm{Fe} / \mathrm{FeF}_{2}$ layers (invited). J. Appl. Phys. 1998, 83, 6893-6895. [CrossRef]

106. Olamit, J.; Li, Z.-P.; Schuller, I.K.; Liu, K. Angular dependence of exchange anisotropy on the cooling field in ferromagnet/fluoride thin films. Phys. Rev. B 2006, 73, 024413. [CrossRef]

107. Velasquez, E.A.; Mazo-Zuluaga, J.; Tangarife, E.; Mejia-Lopez, J. Structural relaxation and crystalline phase effects on the exchange bias phenomenon in $\mathrm{FeF}_{2} / \mathrm{Fe}$ core/shell nanoparticles. Adv. Mater. Interfaces 2020, 7, 2000862. [CrossRef]

108. Tangarife, E.; Romero, A.H.; Mejía-López, J. A charge optimized many-body potential for iron/iron-fluoride systems. Phys. Chem. Chem. Phys. 2019, 21, 20118-20131. [CrossRef]

109. Pechan, M.J.; Bennett, D.; Teng, N.; Leighton, C.; Nogués, J.; Schuller, I.K. Induced anisotropy and positive exchange bias: A temperature, angular, and cooling field study by ferromagnetic resonance. Phys. Rev. B 2002, 65, 064410. [CrossRef]

110. Leighton, C.; Fitzsimmons, M.R.; Hoffmann, A.; Dura, J.; Majkrzak, C.F.; Lund, M.S.; Schuller, I.K. Thickness-dependent coercive mechanisms in exchange-biased bilayers. Phys. Rev. B 2002, 65, 064403. [CrossRef]

111. Arenholz, E.; Liu, K. Angular dependence of the magnetization reversal in exchange-biased Fe/MnF 2. Appl. Phys. Lett. 2005, 87, 132501. [CrossRef]

112. Sudsom, D.; Blachowicz, T.; Hahn, L.; Ehrmann, A. Vortex nucleation and propagation in magnetic double-wedges and semisquares for reliable quaternary storage systems. J. Magn. Magn. Mater. 2020, 514, 167294. [CrossRef]

113. Shameem, P.V.M.; Kumar, M.S. Training effect of the exchange bias in sputter deposited $\mathrm{Fe}_{3} \mathrm{O}_{4}$ thin films with varying thickness. J. Magn. Magn. Mater. 2018, 458, 241-252. [CrossRef]

114. Lee, C.-H.; Anbalagan, A.K.; Chang, T.-W.; Fan, C.-L.; Chung, J.-C.; Chien, S.-C. The exchange bias effect on single layer of Fe-rich FeRh thin film. Mater. Lett. 2019, 254, 309-311. [CrossRef] 
115. Fan, R.; Kinane, C.J.; Charlton, T.R.; Dorner, R.; Ali, M.; de Vries, M.A.; Brydson, R.M.D.; Marrows, C.H.; Hickey, B.J.; Arena, D.A.; et al. Ferromagnetism at the interfaces of antiferromagnetic FeRh epilayers. Phys. Rev. B 2010, 82, 184418. [CrossRef]

116. Mallick, K.; Kumar, P.S.A. Crystallite size dependent exchange bias in $\mathrm{MgFe}_{2} \mathrm{O}_{4}$ thin films on Si(100). J. Appl. Phys. 2018, 124, 053901. [CrossRef]

117. Ling, Y.C.; Liu, R.B.; Wang, H.B.; Xiao, S.Y.; Qu, D.Q.; Wang, G.G.; Han, Z.D.; Du, J.; Xu, Q.Y. Martensitic transformation and large exchange bias in Mn-rich Ni-Mn-Sn thin films on mica substrates. J. Alloys Comp. 2020, 827, 154303. [CrossRef]

118. Khan, M.; Dubenko, I.; Stadler, S.; Ali, N. Exchange bias in bulk Mn rich Ni-Mn-Sn Heusler alloys. J. Appl. Phys. 2007, 102, 113914. [CrossRef]

119. Sánchez Llamazares, J.L.; Flores-Zúniga, H.; Ríos-Jara, D.; Sánchez-Valdes, C.F.; García-Fernández, T.; Ross, C.A.; García, C. Structural and magnetic characterization of the intermartensitic phase transition in NiMnSn Heusler alloy ribbons. J. Appl. Phys. 2013, 113, 17A948. [CrossRef]

120. Giri, S.K.; Sahoo, R.C.; Dasgupta, P.; Poddar, A.; Nath, T.K. Giant spontaneous exchange bias effect in $\mathrm{Sm}_{1.5} \mathrm{Ca}_{0.5} \mathrm{CoMnO}_{6}$ perovskite. J. Phys. D Appl. Phys. 2016, 49, 165002. [CrossRef]

121. Sahoo, R.C.; Das, S.; Giri, S.K.; Paladhi, D.; Nath, T.K. Size modulated Griffiths phase and spin dynamics in double perovskite $\mathrm{Sm}_{1.5} \mathrm{Ca}_{0.5} \mathrm{CoMnO}_{6}$. J. Magn. Magn. Mater. 2019, 469, 161-170. [CrossRef]

122. Panchal, G.; Choudhary, R.J.; Kumar, M.; Phase, D.M. Interfacial spin glass mediated spontaneous exchange bias effect in self-assembled $\mathrm{La}_{0.7} \mathrm{Sr}_{0.3} \mathrm{MnO}_{3}: \mathrm{NiO}$ nanocomposite thin films. J. Alloys Comp. 2019, 796, 196-202. [CrossRef]

123. Wu, Y.J.; Wang, Z.J.; Bai, Y.; Liang, Y.M.; Ning, X.K.; Wang, Q.; Liu, W.; Zhang, Z.D. Transition of the exchange bias effect from in-plane to out-of-plane in $\mathrm{La}_{0.7} \mathrm{Sr}_{0.3} \mathrm{MnO}_{3}: \mathrm{NiO}$ nanocomposite thin films. J. Mater. Chem. C 2019, 7, 6091-6098. [CrossRef]

124. Khadka, D.; Thapaliya, T.R.; Wen, J.J.; Need, R.F.; Huang, S.X. High quality epitaxial thin films and exchange bias of antiferromagnetic Dirac semimetal FeSn. Appl. Phys. Lett. 2020, 117, 032403. [CrossRef]

125. Chen, D.Y.; Chen, Z.H.; He, Q.; Clarkson, J.D.; Serrao, C.R.; Yadav, A.K.; Nowakowski, M.E.; Fan, Z.; You, L.; Gao, X.S.; et al. Interface engineering of domain structures in biFeO 3 thin films. Nano Lett. 2017, 17, 486-493. [CrossRef]

126. Jiang, S.W.; Shan, J.; Mak, K.F. Electric-field switching of two-dimensional van der Waals magnets. Nat. Mater. 2018, 17, 406-410. [CrossRef]

127. Fukami, S.; Zhang, C.L.; DuttaGupta, S.; Kurenkov, A.; Ohno, H. Magnetization switching by spin-orbit torque in an antiferromagnet-ferromagnet bilayer system. Nat. Mater. 2016, 15, 535-541. [CrossRef]

128. Borders, W.A.; Akima, H.; Fukami, S.; Moriya, S.; Kurihara, S.; Horio, Y.; Sato, S.; Ohno, H. Analogue spin-orbit torque device for artificial-neural-network-based associative memory operations. Appl. Phys. Express 2017, 10, 013007. [CrossRef]

129. Polewczyk, V.; Dumesnil, K.; Lacour, D.; Moutaouekkil, M.; Mjahed, H.; Tiercelin, N.; Petit Watelot, S.; Mishra, H.; Dusch, Y.; Hage-Ali, S.; et al. Unipolar and bipolar high-magnetic-field sensors based on surface acoustic wave resonators. Phys. Rev. Appl. 2017, 8, 024001. [CrossRef]

130. Wang, G.P.; Chen, Z.Z.; He, H.C.; Meng, D.C.; Yang, H.; Mao, X.Y.; Pan, Q.; Chu, B.J.; Zuo, M.; Sun, Z.H.; et al. Room temperature exchange bias in structure-modulated single-phase multiferroic materials. Chem. Mater. 2018, 30, 6156-6163. [CrossRef] 